\title{
Vectorlike chiral fourth family to explain muon anomalies
}

\author{
Stuart Raby ${ }^{1, *}$ and Andreas Trautner ${ }^{2, \dagger}$ \\ ${ }^{1}$ Department of Physics, The Ohio State University, \\ 191 W. Woodruff Avenue, Columbus, Ohio 43210, USA \\ ${ }^{2}$ Bethe Center for Theoretical Physics and Physikalisches Institut der Universität Bonn, \\ Nussallee 12, 53115 Bonn, Germany
}

(Received 8 January 2018; published 7 May 2018)

\begin{abstract}
The Standard Model (SM) is amended by one generation of quarks and leptons which are vectorlike (VL) under the SM gauge group but chiral with respect to a new $\mathrm{U}(1)_{3-4}$ gauge symmetry. We show that this model can simultaneously explain the deviation of the muon $g-2$ as well as the observed anomalies in $b \rightarrow s \mu^{+} \mu^{-}$ transitions without conflicting with the data on Higgs decays, lepton flavor violation, or $B_{s}-\bar{B}_{s}$ mixing. The model is string theory motivated and Grand Unified Theory compatible, i.e. UV complete, and fits the data predicting VL quarks, leptons, and a massive $Z^{\prime}$ at the TeV scale, as well as $\tau \rightarrow 3 \mu$ and $\tau \rightarrow \mu \gamma$ within reach of future experiments. The Higgs couplings to SM generations are automatically aligned in flavor space.
\end{abstract}

DOI: 10.1103/PhysRevD.97.095006

\section{INTRODUCTION}

The Standard Model (SM) is a highly successful theory in predicting and fitting many experimental measurements, with few exceptions. One of the discrepancies between the SM prediction and experimental measurement that has been known for a long time is the muon anomalous magnetic moment. The discrepancy between the measured value and the SM prediction is $[1,2]$

$$
\Delta a_{\mu}=a_{\mu}^{\exp }-a_{\mu}^{\mathrm{SM}}=288(63)(49) \times 10^{-11} .
$$

More recently, there appeared deviations from the SM predictions in $b \rightarrow s \mu^{+} \mu^{-}$transitions related to tests of lepton flavor universality in the observables $R(K)$ and $R\left(K^{*}\right)$ [3,4], semileptonic branching ratios [5-7], and angular distributions [8-14]. Most interestingly, all of the more recent anomalies can simultaneously be explained [15-23] by specific deviations from the SM in one or more of the Wilson coefficients $C_{9}, C_{9}^{\prime}, C_{10}$, and/or $C_{10}^{\prime}$ of the effective Hamiltonian [24,25] (see [26] for a possible role of additional tensor operators)

$\mathcal{H}_{\mathrm{eff}}=-\frac{4 G_{\mathrm{F}}}{\sqrt{2}} V_{t b} V_{t s}^{*} \frac{e^{2}}{16 \pi^{2}} \sum_{j=9,10}\left(C_{j} \mathcal{O}_{j}+C_{j}^{\prime} \mathcal{O}_{j}^{\prime}\right)+$ H.c.

\footnotetext{
raby.1@osu.edu

†atrautner@uni-bonn.de
}

Published by the American Physical Society under the terms of the Creative Commons Attribution 4.0 International license. Further distribution of this work must maintain attribution to the author(s) and the published article's title, journal citation, and DOI. Funded by SCOAP. where

$$
\begin{array}{ll}
\mathcal{O}_{9}=\left(\bar{s} \gamma_{\mu} P_{L} b\right)\left(\bar{\mu} \gamma^{\mu} \mu\right), & \mathcal{O}_{9}^{\prime}=\left(\bar{s} \gamma_{\mu} P_{R} b\right)\left(\bar{\mu} \gamma^{\mu} \mu\right), \\
\mathcal{O}_{10}=\left(\bar{s} \gamma_{\mu} P_{L} b\right)\left(\bar{\mu} \gamma^{\mu} \gamma_{5} \mu\right), & \mathcal{O}_{10}^{\prime}=\left(\bar{s} \gamma_{\mu} P_{R} b\right)\left(\bar{\mu} \gamma^{\mu} \gamma_{5} \mu\right) .
\end{array}
$$

A simple extension of the SM that can explain the discrepancy of the muon $g-2$ are vectorlike (VL) leptons that couple exclusively to muons [27-29]. On the other hand, the anomalies in $b \rightarrow s \mu^{+} \mu^{-}$transitions can be explained by a new massive vector boson of a spontaneously broken $U(1)_{\mu-\tau}$ gauge symmetry and the introduction of VL quarks [30,31] (see [32] for a generalization of the new gauge symmetry). Indeed, it has been shown that combining an additional $Z^{\prime}$, VL leptons, and VL quarks one can successfully address both the muon $g-2$ and the anomalous $B$ physics observables simultaneously [33-35]. Typically these models predict significant deviations of the $\mathrm{SM}$ in $h \rightarrow \mu \mu[28,29]$ and $h \rightarrow \mu \tau[31,34]$ and have an upper bound on the $Z^{\prime}$ mass by keeping $B_{s}-\bar{B}_{s}$ oscillations close to their SM value [30].

In the present paper we suggest a holistic way of solving the discrepancies in $(g-2)_{\mu}$ and $b \rightarrow s \mu^{+} \mu^{-}$. We amend the SM by one complete family of fermions, i.e. a full spinor representation of $S O(10)$, which is VL with respect to the SM but chiral with respect to a new spontaneously broken " $U(1)_{3-4}$ " gauge symmetry. Under the new gauge group the third SM family and the left-handed part of the new "VL" family have charges +1 and -1 , respectively, while all other fermions are neutral. Our model is motivated by heterotic string orbifold constructions [36-43], which, in addition to the full Minimal Supersymmetric SM spectrum, typically contain myriads of states which are 
VL with respect to the SM gauge group, but chiral under new $U(1)^{\prime}$ gauge symmetries. In addition, there are many SM singlet scalars that break the additional gauge symmetries, thus giving mass to the vectorlike states and the extra gauge bosons. While in earlier constructions these extra states were lifted to the string scale, our model is a prototype of what can happen if at least one extra generation is kept light, i.e. at the $\mathrm{TeV}$ scale. Our analysis is not supersymmetric, but it could easily be extended to a supersymmetric model in which case gauge coupling unification is maintained.

We find that the model can simultaneously fit the observed quark and lepton masses, as well as the $(g-2)_{\mu}$ and $b \rightarrow s \mu^{+} \mu^{-}$anomalies without violating bounds from electroweak precision observables, lepton flavor violating (LFV) decays, or $B_{s}-\bar{B}_{s}$ mixing. Interestingly, the electroweak singlet Higgs boson couplings in our model are automatically aligned with the SM values to a very high degree. Contrary to $[30,31,34]$ there is no upper bound on the $Z^{\prime}$ mass from the $B_{s}-\bar{B}_{s}$ mixing constraint, simply because the "VL" fermions and the $Z$ ' simultaneously obtain mass of the order of the $U(1)_{3-4}$ breaking scale.

To substantiate our arguments we present two data points that can fit all measured observables while predicting others. The masses of new quarks and leptons, as well as of the new $Z^{\prime}$ are all at the $\mathrm{TeV}$ scale. The $Z^{\prime}$ in our example has very suppressed couplings to the first family, meaning that $Z^{\prime}$ production at the LHC is suppressed. $B_{s}-\bar{B}_{s}$ mixing is predicted to deviate from the SM at the level of a few percent. There are significant enhancements in $\operatorname{BR}\left(B_{s} \rightarrow K^{(*)} \tau \bar{\tau}\right)$ and $\operatorname{BR}\left(B_{s} \rightarrow \phi \tau \bar{\tau}\right)$, while $R_{K^{(*)}}^{\nu \bar{\nu}}$ is suppressed. Furthermore, our best fit points predict $\operatorname{BR}(\tau \rightarrow \mu \gamma)$ and $\operatorname{BR}(\tau \rightarrow 3 \mu)$ in reach of upcoming experiments.

\section{MODEL}

The model under investigation is the SM with three righthanded neutrinos extended by a complete extra generation of left-chiral fields and a complete extra generation of rightchiral fields. Furthermore, we introduce a new " $U(1)_{3-4}$ " gauge symmetry under which the third SM generation as well as the left-chiral part of the fourth generation of particles is charged. The $U(1)_{3-4}$ gauge symmetry is spontaneously broken by the vacuum expectation value (VEV) of the new scalar $\Phi$. All fields and their corresponding quantum numbers are summarized in Table I. The relevant part of the Lagrangian for this study is given by

$\mathcal{L} \supset \mathcal{L}_{3, H}+\mathcal{L}_{\mathrm{VL}, H}+\mathcal{L}_{3, \Phi}+\mathcal{L}_{\mathrm{VL}, \Phi}+\mathcal{L}_{12, \varphi}+\mathcal{L}_{\mathrm{Maj}}$,

with $^{1}$

\footnotetext{
${ }^{1}$ In our notation $P_{L} N_{L}=N_{L}$ is a two component Dirac spinor which can be written in terms of the two component Weyl spinor, $\eta$, as $N_{L}=(\eta, 0)^{\mathrm{T}}$. Then $N_{L}^{\mathcal{C}}=\left(0, \mathrm{i} \sigma_{2} \eta^{*}\right)^{\mathrm{T}}$.
}

$$
\begin{aligned}
\mathcal{L}_{3, H}:= & -y_{b} \bar{q}_{L}^{3} H d_{R}^{3}-y_{\tau} \bar{\ell}_{L}^{3} H e_{R}^{3}-y_{\nu} \bar{\ell}_{L}^{3} \tilde{H} \nu_{R}^{3}+\text { H.c., } \\
\mathcal{L}_{3, \Phi}:= & -\lambda_{3} \Phi\left(\bar{q}_{L}^{3} Q_{R}+\bar{d}_{R}^{3} D_{L}+\bar{\ell}_{L}^{3} L_{R}+\bar{e}_{R}^{3} E_{L}\right)+\text { H.c. } \\
\mathcal{L}_{\mathrm{VL}, H}:= & -\lambda_{L R}\left(\bar{Q}_{L} H D_{R}+\bar{L}_{L} H E_{R}+\bar{L}_{L} \tilde{H} N_{R}\right)+\text { H.c. } \\
& -\lambda_{R L}\left(\bar{Q}_{R} H D_{L}+\bar{L}_{R} H E_{L}+\bar{L}_{R} \tilde{H} N_{L}\right)+\text { H.c., } \\
\mathcal{L}_{\mathrm{VL}, \Phi}:= & -\Phi^{*}\left(\lambda_{Q} \bar{Q}_{L} Q_{R}+\lambda_{D} \bar{D}_{R} D_{L}+\lambda_{L} \bar{L}_{L} L_{R}+\lambda_{E} \bar{E}_{R} E_{L}\right. \\
& \left.+\lambda_{N} \bar{N}_{R} N_{L}\right)+ \text { H.c. }, \\
\mathcal{L}_{12, \varphi}:= & -\lambda_{2} \varphi^{a}\left(\bar{q}_{L}^{a} Q_{R}+\bar{d}_{R}^{a} D_{L}+\bar{\ell}_{L}^{a} L_{R}+\bar{e}_{R}^{a} E_{L}\right)+\text { H.c., } \\
\mathcal{L}_{\mathrm{Maj}}:= & -\frac{1}{2} M_{L} \overline{N_{L}^{c}} N_{L}-\frac{1}{2} M_{R}^{a b} \overline{\left(\nu_{R}^{a}\right)^{\mathcal{C}}} \nu_{R}^{b}-\left(M_{R} \overline{N_{R}^{c}} \nu_{R}^{3}+\text { H.c. }\right) .
\end{aligned}
$$

We take all couplings to be real and-in some Grand Unified Theory (GUT) spirit—set many of them alike. Couplings to the up quark sector electroweak singlets $\left(u_{R}^{a}\right.$, $u_{R}^{3}, U_{R}$, and $U_{L}$ ) are not displayed because they will not be constrained by our analysis. It is summed over the repeated indices $a=1,2$ of the $\mathbf{2} \oplus \mathbf{1}$ flavor structure of the SM families which can, for example, originate from a $\mathrm{D}_{4}$ flavor symmetry $[37,40,42-45]$. The first and second families are distinguished by the direction of the $\mathrm{D}_{4}$ breaking VEV $\left\langle\varphi_{a}\right\rangle=\delta_{a 2} v_{\varphi}$. We assume this alignment to happen at a high-scale $M$ (one should imagine $M \sim M_{\text {string }}$ or $M \sim M_{\mathrm{GUT}}$ ), and the corresponding effective operator coefficient, thus, should be imagined as $v_{\varphi} \equiv$ $\langle\tilde{\Phi}\rangle\left\langle\varphi_{2}\right\rangle / M$ where $\tilde{\Phi}$ is a SM and $\mathrm{U}(1)_{3-4}$ neutral scalar that gets a VEV around the weak scale. This justifies our assumption here that the first family does not directly mix with the VL states. We will focus on the flavor structure of the second and third generations in this study, remarking that the first family can always be fit in. A more detailed analysis should include all three families and their flavor physics, but that is beyond the scope of the present paper.

\section{A. Charged lepton and down quark masses}

The charged lepton mass terms are given by

$$
\overline{\boldsymbol{e}}_{L}^{A} \mathcal{M}_{A B}^{\ell} \boldsymbol{e}_{R}^{B} \equiv\left(\begin{array}{c}
\bar{E}_{L} \\
\bar{E}_{L}^{\prime} \\
\bar{e}_{L}^{3} \\
\bar{e}_{L}^{2}
\end{array}\right)^{\mathrm{T}}\left(\begin{array}{cccc}
\lambda_{R L} v & \lambda_{E} v_{\Phi} & \lambda_{3} v_{\Phi} & \lambda_{2} v_{\varphi} \\
\lambda_{L} v_{\Phi} & \lambda_{L R} v & 0 & 0 \\
\lambda_{3} v_{\Phi} & 0 & y_{\tau} v & 0 \\
\lambda_{2} v_{\varphi} & 0 & 0 & y_{\mu} v
\end{array}\right)\left(\begin{array}{c}
E_{R}^{\prime} \\
E_{R} \\
e_{R}^{3} \\
e_{R}^{2}
\end{array}\right),
$$

where $A, B=1, \ldots, 4$ and the scalar VEVs and couplings are all assumed to be real. Analogously, the down quark mass terms are given by 
TABLE I. The quantum numbers of fermions and scalars in our model under the SM gauge group and under the new $\mathrm{U}(1)^{\prime} \equiv \mathrm{U}(1)_{3-4}$. Note that primed fields have nothing to do with the $\mathrm{U}(1)^{\prime}$ per se but are used to denote constituents of $\mathrm{SU}(2)_{\mathrm{L}}$ doublets.

\begin{tabular}{|c|c|c|c|c|c|c|c|}
\hline$G_{\mathrm{SM}}$ family & $(\mathbf{3}, \mathbf{2})_{\frac{1}{6}}$ & $(\mathbf{3}, \mathbf{1})_{\frac{2}{3}}$ & $(\mathbf{3}, \mathbf{1})_{-\frac{1}{3}}$ & $(\mathbf{1}, \mathbf{2})_{-\frac{1}{2}}$ & $(\mathbf{1}, \mathbf{1})_{-1}$ & $(\mathbf{1}, \mathbf{1})_{0}$ & $\mathrm{U}(1)_{3-4}$ \\
\hline$a=1,2$ & $q_{\mathrm{L}}^{a}=\left(u_{\mathrm{L}}^{a}, d_{\mathrm{L}}^{a}\right)$ & $u_{\mathrm{R}}^{a}$ & $d_{\mathrm{R}}^{a}$ & $\ell_{\mathrm{L}}^{a}=\left(\nu_{\mathrm{L}}^{a}, e_{\mathrm{L}}^{a}\right)$ & $e_{\mathrm{R}}^{a}$ & $\nu_{\mathrm{R}}^{a}$ & 0 \\
\hline 3 & $q_{\mathrm{L}}^{3}=\left(u_{\mathrm{L}}^{3}, d_{\mathrm{L}}^{3}\right)$ & $u_{\mathrm{R}}^{3}$ & $d_{\mathrm{R}}^{\mathrm{n}}$ & $\ell_{\mathrm{L}}^{3}=\left(\nu_{\mathrm{L}}^{3}, e_{\mathrm{L}}^{3}\right)$ & $e_{\mathrm{R}}^{\frac{\pi}{3}}$ & $\nu_{\mathrm{R}}^{\frac{\pi}{3}}$ & 1 \\
\hline $4_{L}$ & $Q_{\mathrm{L}}=\left(U_{\mathrm{L}}^{\prime}, D_{\mathrm{L}}^{\prime}\right)$ & $U_{\mathrm{R}}$ & $D_{\mathrm{R}}$ & $L_{\mathrm{L}}^{\mathrm{L}}=\left(N_{\mathrm{L}}^{\prime}, E_{\mathrm{L}}^{\prime}\right)$ & $E_{\mathrm{R}}$ & $N_{\mathrm{R}}$ & -1 \\
\hline \multirow[t]{2}{*}{$4_{\mathrm{R}}$} & $Q_{\mathrm{R}}=\left(U_{\mathrm{R}}^{\prime}, D_{\mathrm{R}}^{\prime}\right)$ & $U_{\mathrm{L}}$ & $D_{\mathrm{L}}$ & $L_{\mathrm{R}}=\left(N_{\mathrm{R}}^{\prime}, E_{\mathrm{R}}^{\prime}\right)$ & $E_{\mathrm{L}}$ & $N_{\mathrm{L}}$ & 0 \\
\hline & & $H$ & & $\Phi$ & & & $\left(\varphi_{1}, \varphi_{2}\right)$ \\
\hline$G_{\mathrm{SM}}$ & & $(\mathbf{1}, \mathbf{2})_{\frac{1}{2}}$ & & $(\mathbf{1}, \mathbf{1})_{0}$ & & & $(\mathbf{1}, \mathbf{1})_{0}$ \\
\hline $\mathrm{U}(1)_{3-4}$ & & 0 & & 1 & & & 0 \\
\hline $\mathrm{D}_{4}$ & & 1 & & 1 & & & 2 \\
\hline
\end{tabular}

$$
\overline{\boldsymbol{d}}_{L}^{A} \mathcal{M}_{A B}^{d} \boldsymbol{d}_{R}^{B}
$$

with

$$
\begin{aligned}
& \boldsymbol{d}_{L}:=\left(D_{L}, D_{L}^{\prime}, d_{L}^{3}, d_{L}^{2}\right), \\
& \boldsymbol{d}_{R}:=\left(D_{R}^{\prime}, D_{R}, d_{R}^{3}, d_{R}^{2}\right) .
\end{aligned}
$$

The matrix $\mathcal{M}^{d}$ has exactly the same structure as $\mathcal{M}^{\ell}$ with the replacements $\lambda_{E} \rightarrow \lambda_{D}, \lambda_{L} \rightarrow \lambda_{Q}, y_{\tau} \rightarrow y_{b}$, and $y_{\mu} \rightarrow y_{s}$. Let $U_{L}^{\ell, d}$ and $U_{R}^{\ell, d}$ be unitary matrices that diagonalize the respective mass matrix,

$$
\begin{aligned}
& \left(U_{L}^{\ell}\right)^{\dagger} \mathcal{M}^{\ell} U_{R}^{\ell}=\left(\mathcal{M}^{\ell}\right)^{\operatorname{diag}} \equiv \operatorname{diag}\left(m_{E}, m_{L}, m_{\tau}, m_{\mu}\right), \\
& \left(U_{L}^{d}\right)^{\dagger} \mathcal{M}^{d} U_{R}^{d}=\left(\mathcal{M}^{d}\right)^{\operatorname{diag}} \equiv \operatorname{diag}\left(m_{D}, m_{Q}, m_{b}, m_{s}\right) .
\end{aligned}
$$

The physical fields in the mass basis are then given by

$$
\begin{aligned}
{\left[\hat{\boldsymbol{e}}_{L, R}\right]^{A} } & =\left[\left(U_{L, R}^{\ell}\right)^{\dagger}\right]^{A B}\left[\boldsymbol{e}_{L, R}\right]^{B} \\
\text { and } \quad\left[\hat{\boldsymbol{d}}_{L, R}\right]^{A} & =\left[\left(U_{L, R}^{d}\right)^{\dagger}\right]^{A B}\left[\boldsymbol{d}_{L, R}\right]^{B} .
\end{aligned}
$$

\section{B. Neutrino masses}

Defining the vectors

$\nu_{L}:=\left(N_{L}, N_{L}^{\prime}, \nu_{L}^{3}, \nu_{L}^{2}\right)$ and $\nu_{R}:=\left(N_{R}^{\prime}, N_{R}, \nu_{R}^{3}, \nu_{R}^{2}\right)$,

the neutrino masses can be written as

$$
\left(\begin{array}{c}
\overline{\boldsymbol{\nu}}_{L} \\
\overline{\boldsymbol{\nu}}_{R}^{\mathcal{C}}
\end{array}\right)^{\mathrm{T}}\left(\begin{array}{cc}
\mathcal{M}_{L} & \mathcal{M}_{D} \\
\mathcal{M}_{D}^{\mathrm{T}} & \mathcal{M}_{R}
\end{array}\right)\left(\begin{array}{c}
\boldsymbol{\nu}_{L}^{\mathcal{C}} \\
\boldsymbol{\nu}_{R}
\end{array}\right) \equiv \overline{\mathbf{N}}_{L}^{\alpha} \mathcal{M}^{\nu}{ }_{\alpha \beta} \mathbf{N}_{R}^{\beta}
$$

where $\alpha, \beta=1, \ldots, 8$ and $\mathbf{N}_{L}^{\mathcal{C}}=\mathbf{N}_{R}$. The Dirac mass terms $\mathcal{M}_{D}$ have the same structure as $\mathcal{M}^{\ell}$ with the replacements $\lambda_{E} \rightarrow \lambda_{N}, y_{\tau} \rightarrow y_{\nu_{1}}$, and $y_{\mu} \rightarrow y_{\nu_{2}}$. The Majorana mass terms $\mathcal{M}_{L, R}$ have nonzero elements $2\left[\mathcal{M}_{L}\right]_{11}=M_{L}$, $\left[\mathcal{M}_{R}\right]_{23}=\left[\mathcal{M}_{R}\right]_{32}=M_{R}$, and $2\left[\mathcal{M}_{R}\right]_{44}=M_{R}^{11}$ with all other elements being zero. Assuming the hierarchy
$M \sim M_{L, R} \gg v_{\Phi, \varphi} \gg v$ the neutrino mass matrix can be analytically diagonalized, and we give details about that in Appendix A. The physical states are

$$
\hat{\mathbf{N}}_{L}^{\alpha}=\left[\left(U^{\nu}\right)^{\mathrm{T}}\right]^{\alpha \beta} \mathbf{N}_{L}^{\beta}, \quad \hat{\mathbf{N}}_{R}^{\alpha}=\left[\left(U^{\nu}\right)^{\dagger}\right]^{\alpha \beta} \mathbf{N}_{R}^{\beta},
$$

with corresponding masses

$$
\begin{aligned}
\left(U^{\nu}\right)^{\mathrm{T}} \mathcal{M}^{\nu} U^{\nu} & =\left(\mathcal{M}^{\nu}\right)^{\operatorname{diag}} \\
& \approx \operatorname{diag}\left(M, M, \frac{M}{2}, \frac{M}{2}, M_{D}, M_{D}, 0,0\right),
\end{aligned}
$$

up to corrections of the order $v_{(\Phi, \varphi)}^{2} / M$. There are four sterile neutrinos with mass at the high scale. Furthermore, there are two light active neutrinos with mass of order $M_{W}^{2} / M_{\mathrm{GUT}}$ and one (mostly) Dirac neutrino with a TeV scale mass (cf. Appendix A)

$$
M_{D}=\sqrt{\left(\lambda_{L} v_{\Phi}\right)^{2}+\left(\lambda_{3} v_{\Phi}\right)^{2}+\left(\lambda_{2} v_{\varphi}\right)^{2}}
$$

Adding the first generation back in gives one additional high scale sterile neutrino and one additional light active neutrino.

\section{Z-lepton couplings}

The Z-lepton couplings in the mass basis are

$$
\mathcal{L} \supset Z_{\mu}\left(\hat{\overline{\boldsymbol{e}}}_{L}^{A} \gamma^{\mu}\left[\hat{g}_{L}^{Z}\right]_{A B} \hat{\boldsymbol{e}}_{L}^{B}+\hat{\overline{\boldsymbol{e}}}_{R}^{A} \gamma^{\mu}\left[\hat{g}_{R}^{Z}\right]_{A B} \hat{\boldsymbol{e}}_{R}^{B}\right),
$$

with coupling matrices

$$
\hat{g}_{L, R}^{Z}=\left(U_{L, R}^{\ell}\right)^{\dagger} g_{L, R}^{Z} U_{L, R}^{\ell} .
$$

The unhatted coupling matrices are in the gauge basis and given by

$$
g_{L, R}^{Z}=\frac{g}{c_{W}}\left[1 g_{L, R}^{Z, \mathrm{SM}} \pm \operatorname{diag}\left(\frac{1}{2}, 0,0,0\right)\right],
$$


where $g_{L}^{Z, \mathrm{SM}}=\left(-1 / 2+s_{W}^{2}\right), g_{R}^{Z, \mathrm{SM}}=s_{W}^{2}$, and we use the abbreviations $s_{W}=\sin \theta_{W}, c_{W}=\cos \theta_{W}$. Since these matrices are not proportional to the identity matrix, the $Z$-lepton couplings are not diagonal in the mass basis. Hence, this model has LFV $Z$ boson decays, which are, however, only effective amongst the heavy VL quarks and leptons.

\section{D. $W$-lepton couplings}

The $W$-lepton couplings in the mass basis are

$\mathcal{L} \supset W_{\mu}^{+}\left(\hat{\mathbf{N}}_{L}^{\alpha} \gamma^{\mu}\left[\hat{g}_{L}^{W}\right]_{\alpha B} \hat{e}_{L}^{B}+\hat{\mathbf{\mathbf { N }}}_{R}^{\alpha} \gamma^{\mu}\left[\hat{g}_{R}^{W}\right]_{\alpha B} \hat{\boldsymbol{e}}_{R}^{B}\right)+$ H.c.,

where

$$
\begin{aligned}
\hat{g}_{L}^{W} & =\frac{g}{\sqrt{2}}\left[\left(U^{\nu}\right)^{\mathrm{T}} g_{L}^{W} U_{L}^{\ell}\right] \\
\text { and } \quad \hat{g}_{R}^{W} & =\frac{g}{\sqrt{2}}\left[\left(U^{\nu}\right)^{\dagger} g_{R}^{W} U_{R}^{\ell}\right],
\end{aligned}
$$

with the $8 \times 4$ coupling matrices of the gauge basis

$$
\begin{aligned}
g_{L}^{W} & =\left(\begin{array}{c}
\operatorname{diag}(0,1,1,1) \\
\mathbf{0}_{4 \times 4}
\end{array}\right) \\
\text { and } \quad g_{R}^{W} & =\left(\begin{array}{c}
\mathbf{0}_{4 \times 4} \\
\operatorname{diag}(1,0,0,0)
\end{array}\right) .
\end{aligned}
$$

\section{E. $Z^{\prime}$ couplings}

The $Z^{\prime}$ couplings to charged leptons in the mass basis are

$$
\mathcal{L} \supset g^{\prime} Z_{\mu}^{\prime}\left(\hat{\overline{\boldsymbol{e}}}_{L}^{A} \gamma^{\mu}\left[\hat{g}_{L}^{\ell}\right]_{A B} \hat{\boldsymbol{e}}_{L}^{B}+\hat{\overline{\boldsymbol{e}}}_{R}^{A} \gamma^{\mu}\left[\hat{g}_{R}^{\ell}\right]_{A B} \hat{\boldsymbol{e}}_{R}^{B}\right),
$$

where

$$
\hat{g}_{L, R}^{\ell}=\left(U_{L, R}^{\ell}\right)^{\dagger} g_{L, R} U_{L, R}^{\ell},
$$

with the $\mathrm{U}(1)_{3-4}$ charge matrices

$$
g_{L}=g_{R}=\operatorname{diag}(0,-1,1,0) .
$$

The $Z^{\prime}$ couplings here are not left-right symmetric: recall the charge assignment in Table I and our skewed definition of the right-handed states in (13) and (16). The $Z^{\prime}$-down quark couplings in the mass basis are completely analogously given by

$$
\mathcal{L} \supset g^{\prime} Z_{\mu}^{\prime}\left(\hat{\overline{\boldsymbol{d}}}_{L}^{A} \gamma^{\mu}\left[\hat{g}_{L}^{d}\right]_{A B} \hat{\boldsymbol{d}}_{L}^{B}+\hat{\overline{\boldsymbol{d}}}_{R}^{A} \gamma^{\mu}\left[\hat{g}_{R}^{d}\right]_{A B} \hat{\boldsymbol{d}}_{R}^{B}\right),
$$

with

$$
\hat{g}_{L, R}^{d}=\left(U_{L, R}^{d}\right)^{\dagger} g_{L, R} U_{L, R}^{d} .
$$

The $Z^{\prime}$ mediated flavor changing neutral currents (FCNC) between the SM $2 \leftrightarrow 3$ generations are naturally suppressed because they only arise from the mixing with the heavy VL states.

The $Z^{\prime}$ couplings to neutrinos in the mass basis can be written as

$$
\mathcal{L} \supset g^{\prime} Z_{\mu}^{\prime}\left(\hat{\mathbf{N}}_{L}^{\alpha} \gamma^{\mu}\left[\hat{g}^{n}\right]_{\alpha \beta} \hat{\mathbf{N}}_{L}^{\beta}\right),
$$

with the coupling

$$
\hat{g}^{n}=\left(U^{\nu}\right)^{\mathrm{T}} g^{n}\left(U^{\nu}\right)^{*},
$$

and the gauge basis charge matrix

$$
g^{n}=\operatorname{diag}(0,-1,1,0,0,1,-1,0) .
$$

\section{F. Higgs-lepton couplings}

The couplings between the physical Higgs boson, $h$, and the charged leptons in the mass basis are

$$
\mathcal{L} \supset-\frac{1}{\sqrt{2}} h \hat{\overline{\boldsymbol{e}}}_{L}^{A} \hat{Y}_{A B}^{\ell} \hat{\boldsymbol{e}}_{R}^{B}+\text { H.c. }
$$

where

$$
\hat{Y}^{\ell}=\left(U_{L}^{\ell}\right)^{\dagger} Y^{\ell} U_{R}^{\ell},
$$

with the gauge basis couplings

$$
Y^{\ell}=\left(\begin{array}{cccc}
\lambda_{R L} & 0 & 0 & 0 \\
0 & \lambda_{L R} & 0 & 0 \\
0 & 0 & y_{\tau} & 0 \\
0 & 0 & 0 & y_{\mu}
\end{array}\right) .
$$

A very interesting feature of this model is that the masses of the SM families are to a very high accuracy linear in the Higgs VEV. Thus, the Higgs couplings in the mass basis, $\hat{Y}^{\ell}$, are to a high precision diagonal in the lower $2 \times 2$ block. Hence, the Higgs couplings to the SM states are very much SM-like and there are no significant flavor violating Higgs couplings among the SM states. We give an analytic proof of this feature in Appendix B. Flavor off-diagonal couplings of the VL states (also to the SM states) can be sizable.

\section{OBSERVABLES}

\section{A. Lepton nonuniversality}

Generally, our model gives rise to lepton nonuniversality in the operators $\mathcal{O}_{i=9,10}^{(\prime)}$ by tree-level $Z^{\prime}$ exchange. The corresponding effective contributions to the Wilson coefficients are

$$
C_{i}^{(\prime), \mathrm{NP}}=-\frac{\sqrt{2}}{4 G_{\mathrm{F}}} \frac{1}{V_{t b} V_{t s}^{*}} \frac{16 \pi^{2}}{e^{2}} \frac{1}{2 v_{\Phi}^{2}} g_{\mathrm{eff}, i}^{(\prime)}
$$


with the couplings

$$
\begin{array}{ll}
g_{\mathrm{eff}, 9}=\left[\hat{g}_{L}^{d}\right]_{43}\left[\hat{g}_{R}^{\ell}+\hat{g}_{L}^{\ell}\right]_{44}, & g_{\mathrm{eff}, 10}=\left[\hat{g}_{L}^{d}\right]_{43}\left[\hat{g}_{R}^{\ell}-\hat{g}_{L}^{\ell}\right]_{44}, \\
g_{\mathrm{eff}, 9}^{\prime}=\left[\hat{g}_{R}^{d}\right]_{43}\left[\hat{g}_{R}^{\ell}+\hat{g}_{L}^{\ell}\right]_{44}, & g_{\mathrm{eff}, 10}^{\prime}=\left[\hat{g}_{R}^{d}\right]_{43}\left[\hat{g}_{R}^{\ell}-\hat{g}_{L}^{\ell}\right]_{44} .
\end{array}
$$

These couplings are expressible solely through mixing matrix elements, for example,

$$
\begin{aligned}
g_{\mathrm{eff}, 9}= & \left(\left[U_{L}^{d^{\dagger}}\right]_{43}\left[U_{L}^{d}\right]_{33}-\left[U_{L}^{d^{\dagger}}\right]_{42}\left[U_{L}^{d}\right]_{23}\right) \\
& \times\left(\left[U_{L}^{\ell \dagger}\right]_{43}\left[U_{L}^{\ell}\right]_{34}-\left[U_{L}^{\ell \dagger}\right]_{42}\left[U_{L}^{\ell}\right]_{24}\right. \\
& \left.+\left[U_{R}^{\ell \dagger}\right]_{43}\left[U_{R}^{\ell}\right]_{34}-\left[U_{R}^{\ell \dagger}\right]_{42}\left[U_{R}^{\ell}\right]_{24}\right) .
\end{aligned}
$$

While we focus on the $Z^{\prime}$ coupling to muons in order to explain the observed anomalies, our model also modifies the effective Wilson coefficients $C_{9,10}^{(\prime), \tau \tau}$ and $C_{9,10}^{(\prime), \nu \nu}$ leading to lepton nonuniversality also in $\operatorname{BR}\left(B_{s} \rightarrow K^{(*)} \tau \bar{\tau}\right)$ and $\mathrm{BR}\left(B_{s} \rightarrow \phi \tau \bar{\tau}\right)$. Quantitative results for these observables have been obtained using the formulas given in [46] from where we also adopt the SM prediction (cf. also $[47,48])$. The New Physics (NP) contributions to the Wilson coefficients $C_{9,10}^{(\prime), \nu}$ affect the SM prediction for $\operatorname{BR}\left(B_{s} \rightarrow K^{(*)} \nu \bar{\nu}\right)$, and we have followed [49,50] to quantify these effects in our model.

\section{B. Muon anomalous magnetic moment}

The $W, Z$, and $h$ contributions to the anomalous magnetic moment of the muon are very close to their SM values, and we do not detail them here. On the other hand, the $Z^{\prime}$ contribution can be sizable, despite its TeVscale mass. This is due to off-diagonal muon- $Z^{\prime}$ couplings to VL leptons, allowing them to significantly contribute to the $g-2$ loop. Since the VL leptons and $Z^{\prime}$ masses are of the same scale, the leading order contribution has one power of $m_{\mu} / M_{Z^{\prime}}$ less than the naive flavor diagonal $Z^{\prime}$ contribution (cf. e.g. the discussion in [[51], Sec. 7.2]). Parametrically, the dominant modification of $(g-2)_{\mu}$ in our model is of the size

$$
\delta a_{\mu}^{Z^{\prime}} \simeq \frac{m_{\mu}}{16 \pi^{2} v_{\Phi}} \sum_{a \in \mathrm{VL}}\left[\hat{g}_{L}^{\ell}\right]_{4 a}\left[\hat{g}_{R}^{\ell}\right]_{4 a}
$$

where the sum goes over the VL leptons. Naively this points to a scale $v_{\Phi} \sim 10^{2} \mathrm{TeV}$, but the FC couplings to the VL leptons can easily be $\mathcal{O}(0.1)$. In addition, the contributions of individual VL leptons can partly cancel against one another, and we will see this effect to be at work in our numerical analysis below. We give detailed formulas for $\delta a_{\mu}^{Z^{\prime}}$ in Appendix C.

\section{C. $\boldsymbol{B}_{s}-\overline{\boldsymbol{B}}_{s}$ mixing}

There is a new tree-level contribution to $B_{s}-\bar{B}_{s}$ mixing due to $Z^{\prime}$ exchange. We adopt the results and numerical factors from $[30,52]$ and estimate the relative change of the mixing matrix element

$$
\begin{aligned}
\delta M_{12} \simeq & \left(\frac{g^{2}}{16 \pi^{2} M_{W}^{2}}\left(V_{t s} V_{t b}\right)^{2} 2.3\right)^{-1} \\
& \times \frac{1}{2 v_{\Phi}^{2}}\left(\left|\left[\hat{g}_{L}^{d}\right]_{34}\right|^{2}+\left|\left[\hat{g}_{R}^{d}\right]_{34}\right|^{2}+9.7 \operatorname{Re}\left(\left[\hat{g}_{L}^{d}\right]_{34}\left[\hat{g}_{R}^{d, *}\right]_{34}\right)\right) .
\end{aligned}
$$

The most recently updated theoretical uncertainty shows that a deviation from the SM of $\delta M_{12} \lesssim 6 \%$ can currently not be excluded [53]. This gives an important constraint on the down sector flavor changing $Z^{\prime}$ couplings. In our model the $b-s$ coupling is suppressed because it only arises from the mixing with the heavy VL states such that the $Z^{\prime}$ can be kept at the $\mathrm{TeV}$ scale consistent with the bound derived in [54].

\section{Lepton flavor violating $\tau$ decays}

Tree-level $Z^{\prime}$ exchange also induces the decay $\tau \rightarrow 3 \mu$. We follow Refs. [55,56] to estimate

$$
\begin{aligned}
\operatorname{BR}(\tau \rightarrow 3 \mu) \approx & \frac{1}{\Gamma_{\tau}} \frac{m_{\tau}^{5}}{1536 \pi^{3}} \frac{1}{4 v_{\Phi}^{4}} \\
& \times\left(2\left|\left[\hat{g}_{L}^{\ell}\right]_{43}\left[\hat{g}_{L}^{\ell}\right]_{44}\right|^{2}+2\left|\left[\hat{g}_{R}^{\ell}\right]_{43}\left[\hat{g}_{R}^{\ell}\right]_{44}\right|^{2}\right. \\
& \left.+\left|\left[\hat{g}_{L}^{\ell}\right]_{43}\left[\hat{g}_{R}^{\ell}\right]_{44}\right|^{2}+\left|\left[\hat{g}_{R}^{\ell}\right]_{43}\left[\hat{g}_{L}^{\ell}\right]_{44}\right|^{2}\right) .
\end{aligned}
$$

There is also a new contribution to $\tau \rightarrow \mu \gamma$ due to $Z^{\prime}$ and the new VL leptons in the loop, which is enhanced by a power of the VL mass. Using the results of [57] (cf. also [58-60]) we estimate the leading order contribution to be

$$
\begin{aligned}
\operatorname{BR}(\tau \rightarrow \mu \gamma) \simeq & \frac{1}{\Gamma_{\tau}} \frac{\alpha m_{\tau}^{3}}{1024 \pi^{4}} \frac{1}{4 v_{\Phi}^{2}}\left\{\left|\sum_{a \in \mathrm{VL}}\left[\hat{g}_{L}^{\ell}\right]_{4 a}\left[\hat{g}_{R}^{\ell}\right]_{a 3}\right|^{2}\right. \\
& \left.+\left|\sum_{a \in \mathrm{VL}}\left[\hat{g}_{R}^{\ell}\right]_{4 a}\left[\hat{g}_{L}^{\ell}\right]_{a 3}\right|^{2}\right\},
\end{aligned}
$$

where the sum is over internal VL leptons. For the numerical analysis we have used a more detailed result which we present in Appendix D.

\section{E. Other observables}

Flavor violating couplings of the $Z$ or the SM scalar $h$ to the SM families are generally suppressed far below their experimental thresholds. In contrast, flavor changing couplings to the heavy VL leptons can be large. Since our $Z^{\prime}$ is heavy, neutrino trident production $[30,61]$ does not give any important constraints. Lepton unitarity bounds (cf. e.g. $[62,63])$ are easily fulfilled. In addition, there are no 
constraints coming from the branching ratios for $h \rightarrow \gamma \gamma$ or $h \rightarrow g g$ via loop diagrams, since these contributions are suppressed by factors of $\left(v / M_{V L}\right)^{2}$ (see e.g. [59,64-66]).

\section{ANALYSIS}

\section{A. Strategy}

This model can explain the anomalies in the muon $g-2$ and $b \rightarrow s \mu^{+} \mu^{-}$transitions without obviously conflicting with other experimental data. To demonstrate this, we have constructed a $\chi^{2}$-function including the appropriate errors to simultaneously fit the anomaly in $(g-2)_{\mu}$ with the value given in (1), and to reproduce two of the best-fit values of [15] (cf. also [16-23]) for a consistent explanation of $b \rightarrow s \mu^{+} \mu^{-}$anomalies:

$$
\begin{aligned}
&(\mathrm{I}): C_{9}^{\mathrm{NP}} \approx-1.21 \pm 0.2, \quad C_{9}^{\prime} \approx C_{10}^{\mathrm{NP}} \approx C_{10}^{\prime} \approx 0, \\
& \text { or } \quad(\mathrm{II}): C_{9}^{\mathrm{NP}} \approx-1.25 \pm 0.2, \quad C_{9}^{\prime} \approx 0.59 \pm 0.2, \\
& C_{10}^{\mathrm{NP}} \approx C_{10}^{\prime} \approx 0 .
\end{aligned}
$$

Furthermore we fit the masses $m_{d}, m_{s}, m_{\mu}$, and $m_{\tau}$ at the weak scale [67] while requiring $\delta M_{12} \lesssim 15 \%$ to be consistent with the data and theoretical error of $B_{s}-\bar{B}_{s}$ mixing. All other observables are not constrained in the fit; i.e. they arise as predictions of our best fit points. However, there are currently more parameters than observables, and so it is not excluded that there are more points that fit the data well with different predictions. The first family could always be included in the analysis in a straightforward way without affecting our conclusions. ${ }^{2}$

\section{B. Results}

We have found two points which give a very good fit for the cases (I.) and (II.), and they are listed in Table II (and in Table IV in Appendix E). We cannot find a good fit for $C_{9}^{\mathrm{NP}} \simeq-C_{10}^{\mathrm{NP}}$.

The predictions of the best fit points are listed in Table III together with current experimental bounds. Effects on other observables such as $h \rightarrow \mu \tau, h \rightarrow \gamma \gamma, h \rightarrow g g$, neutrino trident production, or Pontecorvo-Maki-Nakagawa-Sakata matrix (PMNS) unitarity violation have also been considered, but they are robustly suppressed in this model and so

\footnotetext{
${ }^{2}$ Including the first family in the most straightforward way the Yukawa couplings and hence resulting mass matrices are extended to (and similarly for neutrinos and down quarks)

$$
\mathcal{M}^{\ell}=\left(\begin{array}{ccccc}
\lambda_{R L} v & \lambda_{E} v_{\Phi} & \lambda_{3} v_{\Phi} & \lambda_{2} v_{\varphi} & 0 \\
\lambda_{L} v_{\Phi} & \lambda_{L R} v & 0 & 0 & 0 \\
\lambda_{3} v_{\Phi} & 0 & y_{\tau} v & 0 & 0 \\
\lambda_{2} v_{\varphi} & 0 & 0 & y_{22} v & y_{21} v \\
0 & 0 & 0 & y_{12} v & y_{11} v
\end{array}\right)
$$
}

TABLE II. Best fit point to the data. We do not list $M_{R}, \lambda_{\nu 1,2}$, and $\lambda_{N}$ because their precise values do not affect the results.

\begin{tabular}{ll}
\hline \hline \multicolumn{2}{c}{ Best fit point (I.) } \\
\hline$\lambda_{\mu}=-0.00008520346$ & $\lambda_{\tau}=0.010031941$ \\
$\lambda_{s}=-0.002313419$ & $\lambda_{b}=-0.01639676$ \\
$\lambda_{L}=0.8595483$ & $\lambda_{E}=0.8596570$ \\
$\lambda_{D}=-1.7819031$ & $\lambda_{Q}=-0.3253384$ \\
$\lambda_{3}=-0.018705093$ & $\lambda_{2}=-0.7701059$ \\
$\lambda_{R L}=-0.9926161$ & $\lambda_{L R}=0.0014518601$ \\
$v_{\varphi}=1603.0788$ & $v_{\phi}=1622.5729$ \\
$g^{\prime}=-0.7518533$ & \\
\hline \hline
\end{tabular}

we do not discuss them in detail. The explicit couplings of the $Z$ boson are stated in Appendix F. While $Z \rightarrow \mu \tau$ is practically absent at tree level, the dominant contribution arises from a one-loop diagram involving $Z^{\prime}$ and $\mathrm{VL}$ leptons in the loop. A rough estimate of this shows that $\operatorname{BR}(Z \rightarrow \mu \tau)$ nonetheless comes out well below the current bound of $1.2 \times 10^{-5}$ [2] (see e.g. [62,68-70] for more discussions of FCNCs in the context of VL fermions).

The loop contributions to $(g-2)_{\mu}$ from $Z, W$, and $h$ are very close to their SM values while the $\mathrm{FC} Z^{\prime}$ exchange with VL leptons in the loop completely accounts for the anomaly. The $Z^{\prime}$ contribution to $B_{s}-\bar{B}_{s}$ is mixing suppressed while larger flavor diagonal couplings to $\mu \bar{\mu}$ can explain a sizable $C_{9}^{\mathrm{NP}}$. The fit prefers a corner of the parameter space where $\lambda_{E} \sim \lambda_{L}$ which makes the $Z^{\prime}$ couplings to leptons approximately left-right (anti)symmetric [the couplings are left-right (LR) symmetric or LR antisymmetric depending on the specific coupling, and we display the coupling matrices for one of the best fit points in Appendix E]. The approximately LR symmetric couplings to the mu and tau leptons lead to an enhanced contribution to $C_{9}^{\mathrm{NP}}$ (and possibly also $C_{9}^{\prime}$ ) and a dramatic cancellation in the axial vector couplings to leptons $C_{10}^{(\prime)}$. By contrast, the approximately equal but opposite LR (anti)symmetric contributions of individual VL leptons to the muon $g-2$ and $\tau \rightarrow \mu \gamma$ cancel only to an order of magnitude. There are no cancellations in $\tau \rightarrow 3 \mu$ where the couplings, in fact, add up constructively. Nevertheless, the tree level process $\tau \rightarrow 3 \mu$ is suppressed by the naturally small flavor offdiagonal couplings of the $Z^{\prime}$ to the SM fermion generations. The best-fit predictions for $\operatorname{BR}(\tau \rightarrow \mu \gamma)$ and $\operatorname{BR}(\tau \rightarrow 3 \mu)$ fall close to regions that can be probed by future experiments [78]; cf. Fig. 1(a). A positive value of $C_{9}^{\prime}$ allows for a negative shift of $\delta M_{12}$, thereby cushioning a $1.8 \sigma$ tension between SM and experiment [53]. This happens to be the case for our best fit point (II.).

The NP contributions to the Wilson coefficients $C_{9,10}^{(\prime), \tau}$ qualitatively follow the patterns in (51) but with reversed signs. $\operatorname{BR}\left(B_{s} \rightarrow K \tau \bar{\tau}\right)$ and $\operatorname{BR}\left(B_{s} \rightarrow \phi \tau \bar{\tau}\right)$ are significantly increased compared to the SM; cf. Fig. 1(b). By contrast $R_{K^{(*)}}^{\nu \bar{v}}$ is suppressed compared to the SM. The suppression of 
TABLE III. Values of (unfitted) observables at our best fit points (central values) and corresponding bounds.

\begin{tabular}{lccc}
\hline \hline Observable & Best Fit (I.) & Best Fit (II.) & Bound \\
\hline$m_{L}, m_{E}$ & $1.78 \mathrm{TeV}, 1.95 \mathrm{TeV}$ & $2.08 \mathrm{TeV}, 2.25 \mathrm{TeV}$ & $>450 \mathrm{GeV}[71]$ \\
$m_{Q}, m_{D}$ & $1.34 \mathrm{TeV}, 3.15 \mathrm{TeV}$ & $1.61 \mathrm{TeV}, 3.49 \mathrm{TeV}$ & $>900 \mathrm{GeV}[72]$ \\
$M_{D}$ & $1.86 \mathrm{TeV}$ & $2.16 \mathrm{TeV}$ & \\
$M_{Z^{\prime}}$ & $1.73 \mathrm{TeV}$ & $2.25 \mathrm{TeV}$ & $<4.4 \times 10^{-8}[73]$ \\
$\tau \rightarrow \mu \gamma$ & $4.6 \times 10^{-9}$ & $5.3 \times 10^{-9}$ & $<2.1 \times 10^{-8}[74]$ \\
$\tau \rightarrow 3 \mu$ & $6.7 \times 10^{-10}$ & $1.1 \times 10^{-9}$ & $\lesssim \pm 6 \%[53]$ \\
$\delta M_{12}\left(B_{s}-\bar{B}_{s}\right)$ & $1 \%$ & $-3 \%$ & $<2.25 \times 10^{-3}[75]$ \\
$\operatorname{BR}\left(B_{s} \rightarrow K \tau \bar{\tau}\right)^{[15,22]}$ & $1.8 \times 10^{-7}$ & $1.4 \times 10^{-7}$ & \\
$\operatorname{BR}\left(B_{s} \rightarrow K^{*} \tau \bar{\tau}\right)^{[15,19]}$ & $2.5 \times 10^{-7}$ & $2.9 \times 10^{-7}$ & $<4.3[76]$ \\
$\operatorname{BR}\left(B_{s} \rightarrow \phi \tau \bar{\tau}\right)^{[15,18.8]}$ & $2.2 \times 10^{-7}$ & $2.6 \times 10^{-7}$ & $<4.4[77]$ \\
$R_{K}^{\nu \bar{\nu}}$ & 0.91 & 0.93 & 0.93 \\
$R_{K^{\nu \nu}}$ & 0.91 & & $<$ \\
\hline \hline
\end{tabular}

$R_{K^{(*)}}^{\nu \bar{\nu}}$ together with a $Z^{\prime}$ explanation of $b \rightarrow s \mu^{+} \mu^{-}$is not in contradiction with the results of [49]. In our model, we find that the enhancement of $R_{K^{(*)}}^{\nu \bar{v}}$ from the second family [49] is counteracted by a vast suppression of the third family contributions.

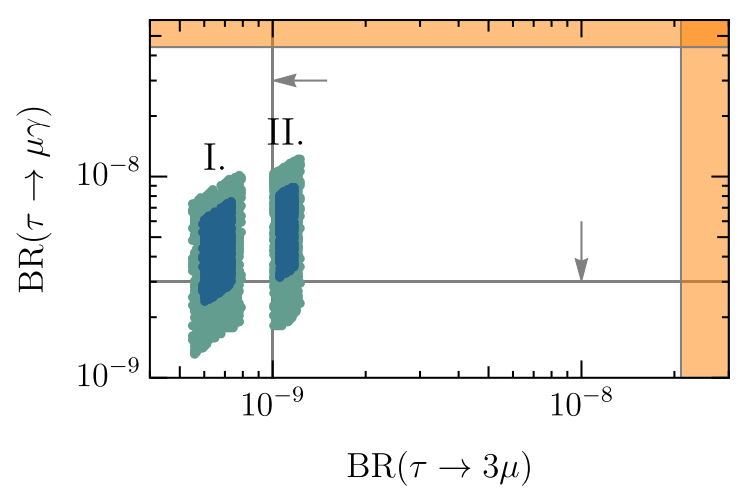

(a)

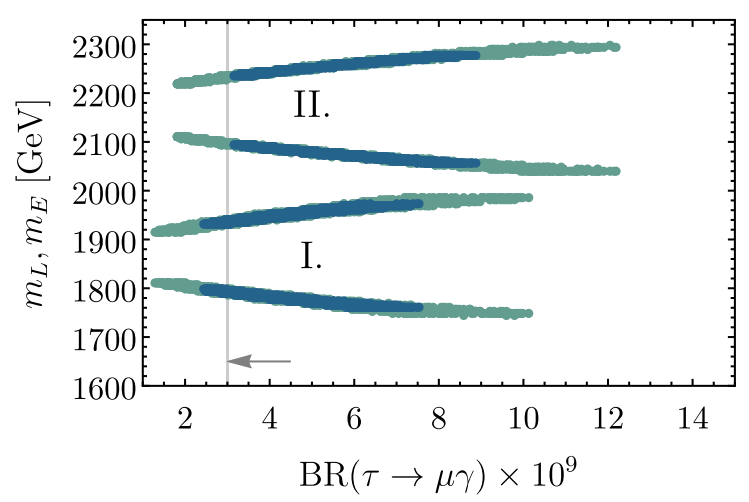

(c)
We do not find any observable that causes problems. We stress again that the $Z^{\prime}$ does, by construction, only very feebly couple to the SM first generation. The gauge coupling $g^{\prime}$ is only very mildly constrained by the fit with a $\chi^{2}<3$ region of $g^{\prime} \in-[5.33(5.08), 0.32(0.39)]$ for

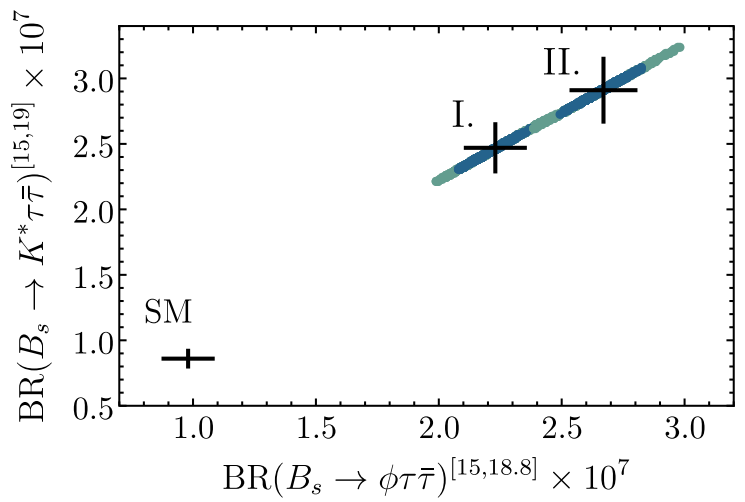

(b)

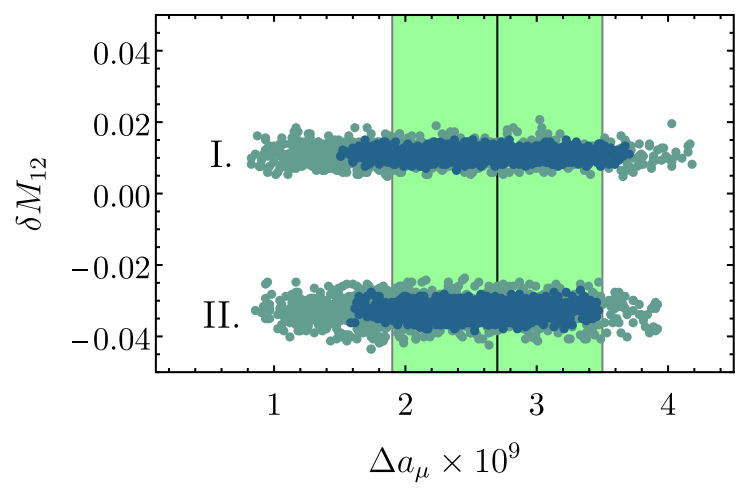

(d)

FIG. 1. The dots show random events scattered in the $\chi^{2}<1(3)$ regions around our best fit points (I.) (Table II) and (II.) (Table IV). The colored regions and gray lines in (a) and (c) show the current experimental exclusion [73,74] and prospects [78], respectively. The lower cross in (b) denotes the SM expectation [46] while the upper crosses show our best fit points with errors computed as in [46]. The green area in (d) shows the best fit value and errors as in Eq. (1). 
TABLE IV. Best fit point to the data for case (II.). We do not list $M_{R}, \lambda_{\nu 1,2}$, and $\lambda_{N}$ because their precise values do not affect the results.

\begin{tabular}{ll}
\hline \hline \multicolumn{2}{c}{ Best fit point (II.) } \\
\hline$\lambda_{\mu}=-0.00023171652$ & $\lambda_{\tau}=0.010033469$ \\
$\lambda_{s}=-0.0009612744$ & $\lambda_{b}=-0.016371133$ \\
$\lambda_{L}=0.8806972$ & $\lambda_{E}=0.8835616$ \\
$\lambda_{D}=-1.8024639$ & $\lambda_{Q}=-0.2965286$ \\
$\lambda_{3}=-0.02561451$ & $\lambda_{2}=-0.8882496$ \\
$\lambda_{R L}=-1.021554$ & $\lambda_{L R}=0.0014201883$ \\
$v_{\varphi}=1717.639$ & $v_{\phi}=1738.4050$ \\
$g^{\prime}=-0.9171299$ & \\
\hline \hline
\end{tabular}

point (I.)[(II.)]. Bounds for family specific $Z^{\prime}$ bosons can be obtained from the LHC $\sqrt{s}=13 \mathrm{TeV}$ searches [79,80] and range from $M_{Z^{\prime}} \gtrsim 1.3$ to $2.0 \mathrm{TeV}$ [81,82], while some models can survive with $M_{Z^{\prime}}$ as low as $\gtrsim 500 \mathrm{GeV}$ [83]. Deriving a robust limit for our model requires one to specify the details of the first family couplings which is beyond the scope of this work. Nevertheless, our $Z^{\prime}$ mass comes out right in the ballpark of current limits, and so it can be searched for at the LHC in dimuon final states. If not with the full run 2 data, our best fit parameter regions will conclusively be tested by a high-luminosity run of the LHC.

We have scattered 1000 points randomly within the $\chi^{2}<1(3)$ regions around the best fit values. There are strong correlations among the masses of the pair of VL leptons, and between the VL lepton masses and the prediction for $\operatorname{BR}(\tau \rightarrow \mu \gamma)$ in Fig. 1(c). Also $\operatorname{BR}\left(B_{s} \rightarrow\right.$ $K \tau \bar{\tau})$ and $\operatorname{BR}\left(B_{s} \rightarrow \phi \tau \bar{\tau}\right)$ are strongly correlated in Fig. 1(b). All other predicted observables are largely uncorrelated; see e.g. Fig. 1(d).

Given the parameters of our best fit points we can also evaluate the effective Higgs couplings of the SM quarks and leptons (see Appendix B for details). The resulting Higgs couplings are diagonal in the mass basis and, moreover, proportional to the masses, just as in the Standard Model.

For model building it is interesting to compare the (gauge basis) Higgs Yukawa couplings to the mixing induced contributions to masses of the SM fermion generations. The muon and strange quark obtain mass predominantly due to mixing effects, and this is also reflected by their large off-diagonal couplings to the VL states. The tau lepton and $b$ quark, by contrast, obtain almost all of their mass from the direct Higgs Yukawa coupling, and mixing effects are miniscule.

\section{CONCLUSION}

We have studied the Standard Model extended by one complete family of "VL" fermions, including right-handed neutrinos, which are vectorlike with respect to the Standard
Model gauge group. In addition, we have introduced a new "U(1) ${ }_{3-4}$ " gauge symmetry under which the third SM family of quarks and leptons have $\mathrm{U}(1)_{3-4}$ charge 1 while the left-chiral part of the new fourth family has charge -1 . Hence, the model is free of gauge anomalies. In our analysis, we have only considered the mixing of the VL states with the second and third SM families, while the absence of mixing to the first family is motivated by a high scale flavor symmetry. The first family could always be included in a straightforward way without affecting our conclusions, and this should be done in the future in order to study the collider phenomenology of this model in more detail.

We have shown that this model can fit the anomalies in the muon $g-2$ and $b \rightarrow s \mu^{+} \mu^{-}$transitions without conflicting with other experimental data. The best fit points predict (cf. Table III) new quarks, leptons, and a family specific $Z^{\prime}$ at the $\mathrm{TeV}$ scale, as well as testable effects in the lepton flavor violating processes $\tau \rightarrow \mu \gamma$ and $\tau \rightarrow 3 \mu$. Furthermore, we find a significant enhancement of $\operatorname{BR}\left(B_{s} \rightarrow K^{(*)} \tau \bar{\tau}\right)$ and $\operatorname{BR}\left(B_{s} \rightarrow \phi \tau \bar{\tau}\right)$, while $R_{K^{(*)}}^{\nu \bar{\nu}}$ is suppressed relative to the SM. Effects on other observables such as $Z \rightarrow \mu \tau, h \rightarrow \mu \tau, h \rightarrow \gamma \gamma, h \rightarrow g g$, neutrino trident production, or PMNS unitarity violation are all robustly suppressed. The Higgs couplings in our model are to a very high degree SM-like, and we have given an analytic proof of that.

\section{ACKNOWLEDGMENTS}

A. T. is grateful to J. Heeck, M.E. Krauss, and A. Vicente for useful discussions. S. R. acknowledges that this material is based upon work supported by the Department of Energy under Award No. DE-SC0011726. This work has partially been supported by the German Science Foundation (DFG) within the SFB-Transregio TR33 "The Dark Universe." S. R. acknowledges partial support from the Alexander von Humboldt Foundation and the Bethe Center of Bonn University where this work was begun, and the Kobayashi-Maskawa Institute, Nagoya University where this work was completed.

\section{APPENDIX A: NEUTRINO SEESAW}

The neutrino mass matrix is described in (21). In the limit $M \sim M_{L, R} \gg v_{\Phi, \varphi} \gg v$ the eigenvectors of $\mathcal{M}^{\nu}$ are to a very good approximation given by

$$
\begin{aligned}
& N_{1,2}=\left(0,0,0,0,0, \frac{1}{\sqrt{2}}, \pm \frac{1}{\sqrt{2}}, 0\right), \\
& N_{3,4}=\left(\frac{1}{\sqrt{2}}, 0,0,0,0,0,0, \pm \frac{1}{\sqrt{2}}\right), \\
& N_{5,6}=\frac{1}{\sqrt{2}}\left(0, \frac{\lambda_{L} v_{\Phi}}{M_{D}}, \frac{\lambda_{3} v_{\Phi}}{M_{D}}, \frac{\lambda_{2} v_{\varphi}}{M_{D}}, \pm 1,0,0,0\right),
\end{aligned}
$$


$N_{7}=\left(0, \frac{\lambda_{3}}{\sqrt{\lambda_{3}^{2}+\lambda_{L}^{2}}},-\frac{\lambda_{L}}{\sqrt{\lambda_{3}^{2}+\lambda_{L}^{2}}}, 0,0,0,0,0\right)$,

$N_{8}=\frac{1}{\mathcal{N}_{8}}\left(0,1,-\frac{\lambda_{3}}{\lambda_{L}},-\frac{v_{\Phi}}{v_{\varphi}} \frac{\left(\lambda_{3}^{2}+\lambda_{L}^{2}\right)}{\lambda_{2} \lambda_{L}}, 0,0,0,0\right)$.

Here we have used

$$
\begin{aligned}
M_{D} & :=\sqrt{\left(\lambda_{L} v_{\Phi}\right)^{2}+\left(\lambda_{3} v_{\Phi}\right)^{2}+\left(\lambda_{2} v_{\varphi}\right)^{2}} \\
\mathcal{N}_{8} & :=\sqrt{1+\left(\frac{\lambda_{3}}{\lambda_{L}}\right)^{2}+\left[\frac{v_{\Phi}}{v_{\varphi}} \frac{\left(\lambda_{3}^{2}+\lambda_{L}^{2}\right)}{\lambda_{2} \lambda_{L}}\right]^{2}} .
\end{aligned}
$$

It is then straightforward to construct the diagonalization matrix $U^{\nu}$ from the eigenvectors.

\section{APPENDIX B: HIGGS COUPLING DIAGONALIZATION}

We wish to show that the Higgs couplings (40) are diagonal in the mass basis. We will focus on the charged leptons here, but the whole analysis of this section fully applies also to the $d$-type quarks by formally replacing $\lambda_{E} \rightarrow \lambda_{D}, \lambda_{L} \rightarrow \lambda_{Q}, \quad y_{\tau} \rightarrow y_{b}$, and $y_{\mu} \rightarrow y_{s}$. Phenomenologically we are led to the relations $v \ll v_{\Phi} \sim$ $v_{\varphi}$ and $\lambda_{L R}, y_{\tau}, y_{\mu} \ll 1$. Therefore, we will treat the lower $3 \times 3$ block of $\mathcal{M}^{\ell}$ in (13) as perturbation. The leading order mass matrix, hence, is given by

$$
\mathcal{M}^{\ell} \approx \widetilde{\mathcal{M}}^{\ell} \equiv v_{\Phi}\left(\begin{array}{cccc}
\lambda_{R L} \xi & \lambda_{E} & \lambda_{3} & \lambda_{2} \\
\lambda_{L} & 0 & 0 & 0 \\
\lambda_{3} & 0 & 0 & 0 \\
\lambda_{2} & 0 & 0 & 0
\end{array}\right),
$$

where $\xi:=v / v_{\Phi}$. The left- and right-singular vectors of this matrix are given by

$$
\begin{gathered}
u_{L, 1}^{\ell}=\frac{1}{N_{L, 1}^{1 / 2}}\left(-u_{-}^{L}, \frac{\lambda_{L}}{\lambda_{2}}, \frac{\lambda_{3}}{\lambda_{2}}, 1\right), \\
u_{R, 1}^{\ell}=\frac{1}{N_{R, 1}^{1 / 2}}\left(u_{-}^{R},-\frac{\lambda_{E}}{\lambda_{2}},-\frac{\lambda_{3}}{\lambda_{2}},-1\right), \\
u_{L, 2}^{\ell}=\frac{1}{N_{L, 2}^{1 / 2}}\left(u_{+}^{L},-\frac{\lambda_{L}}{\lambda_{2}},-\frac{\lambda_{3}}{\lambda_{2}},-1\right), \\
u_{R, 2}^{\ell}=\frac{1}{N_{R, 2}^{1 / 2}}\left(u_{+}^{R},-\frac{\lambda_{E}}{\lambda_{2}},-\frac{\lambda_{3}}{\lambda_{2}},-1\right),
\end{gathered}
$$

where

$$
u_{\mp}^{L(R)}:=\frac{(-)\left(\lambda_{L}^{2}-\lambda_{E}^{2}\right)-\xi^{2} \lambda_{R L}^{2} \mp \sqrt{\left(\lambda_{E}^{2}-\lambda_{L}^{2}\right)^{2}+4 \xi^{2} \lambda_{2}^{2} \lambda_{R L}^{2}+4 \xi^{2} \lambda_{3}^{2} \lambda_{R L}^{2}+2 \xi^{2} \lambda_{E} \lambda_{R L}^{2}+2 \xi^{2} \lambda_{L}^{2} \lambda_{R L}^{2}+\xi^{4} \lambda_{R L}^{4}}}{2 \xi \lambda_{2} \lambda_{R L}},
$$

and the normalization factors $N_{L(R), i}$ are defined by the requirement $\left|u_{L(R), i}^{\ell}\right|^{2}=1$. The matrices $\tilde{U}_{L, R}^{\ell}$ have these vectors as columns. The four corresponding singular values are given by

$$
\left(\tilde{U}_{L}^{\ell}\right)^{\dagger} \widetilde{\mathcal{M}}^{\ell} \tilde{U}_{R}^{\ell}=\operatorname{diag}\left(\tilde{m}_{E}, \tilde{m}_{L}, 0,0\right),
$$

where one can obtain analytic expressions for $\tilde{m}_{E, L}$ but we do not need them here and so they are not displayed. Numerically, $\tilde{U}_{L, R}^{\ell} \approx U_{L, R}^{\ell}$ and $\tilde{m}_{E} \approx m_{E}$ and $\tilde{m}_{L} \approx m_{L}$ hold at the percent level. Now regard the perturbation

$$
\mathcal{M}^{\ell}=\widetilde{\mathcal{M}}^{\ell}+\xi \operatorname{diag}\left(0, \lambda_{L R}, y_{\tau}, y_{\mu}\right)
$$

To leading order in perturbation theory the eigenvalues of the perturbed matrix are obtained by diagonalizing it with the zeroth-order left- and right-singular matrices. Following this procedure we find the small singular values of $\mathcal{M}^{\ell}$ to be

$\tilde{m}_{\tau}=\frac{v}{N_{3}}\left|\lambda_{\tau}+\frac{\lambda_{3}^{2} \lambda_{L R}}{\lambda_{E} \lambda_{L}}\right|$ and $\tilde{m}_{\mu}=\frac{v}{N_{2}}\left|\lambda_{\mu}+\frac{\lambda_{2}^{2} \lambda_{L R}}{\lambda_{E} \lambda_{L}}\right|$

with 


$$
N_{2(3)}:=\sqrt{\left[1+\left(\lambda_{2(3)} / \lambda_{E}\right)^{2}\right]\left[1+\left(\lambda_{2(3)} / \lambda_{L}\right)^{2}\right]}
$$

These values agree with the numerical values to $\mathcal{O}(1 \%)$. There are off-diagonal residuals which are given by

$$
\begin{aligned}
& {\left[\left(\tilde{U}_{L}^{\ell}\right)^{\dagger} \mathcal{M}^{\ell} \tilde{U}_{R}^{\ell}\right]_{34}=\delta m_{\tau \mu} \equiv \frac{v \lambda_{2} \lambda_{3} \lambda_{L R}}{\sqrt{\left(\lambda_{2}^{2}+\lambda_{E}^{2}\right)\left(\lambda_{3}^{2}+\lambda_{L}^{2}\right)}},} \\
& {\left[\left(\tilde{U}_{L}^{\ell}\right)^{\dagger} \mathcal{M}^{\ell} \tilde{U}_{R}^{\ell}\right]_{43}=\delta m_{\mu \tau} \equiv \frac{v \lambda_{2} \lambda_{3} \lambda_{L R}}{\sqrt{\left(\lambda_{3}^{2}+\lambda_{E}^{2}\right)\left(\lambda_{2}^{2}+\lambda_{L}^{2}\right)}} .}
\end{aligned}
$$

They are numerically subdominant compared to the diagonal entries.

The crucial point is that the Higgs couplings in the gauge basis (41) are, at least with regard to the lower $2 \times 2$ block, exactly of the same form as the perturbations to the mass matrix. Approximately diagonalizing the mass matrices with $\tilde{U}_{L, R}^{\ell}$, hence, results in the Higgs couplings

$$
\tilde{Y}_{\text {light }}^{\ell}=\frac{1}{v}\left(\begin{array}{cc}
\tilde{m}_{\tau} & \delta m_{\tau \mu} \\
\delta m_{\mu \tau} & \tilde{m}_{\mu}
\end{array}\right),
$$

which are directly proportional to the mass matrices. There are slight deviations to the proportionality arising at higher order, but they are numerically not relevant.

Evaluating the effective Higgs couplings in the mass basis numerically for our best-fit point (I.) (cf. Table II) one finds

$$
\begin{aligned}
& \hat{Y}_{\text {light }}^{\ell}=\frac{\mathrm{GeV}}{v}\left(\begin{array}{cc}
1.74618 & 3.46843 \times 10^{-7} \\
3.47055 \times 10^{-7} & 0.102717
\end{array}\right) . \\
& \hat{Y}_{\text {light }}^{d}=\frac{\mathrm{GeV}}{v}\left(\begin{array}{cc}
2.85391 & 7.71892 \times 10^{-7} \\
1.41639 \times 10^{-6} & 0.0543716
\end{array}\right) .
\end{aligned}
$$

Clearly the off-diagonal corrections are negligible. Thus, the Higgs coupling to quarks and leptons is diagonal in the mass basis and, moreover, proportional to the masses, just as in the Standard Model.

\section{Integrating out the VL fermions}

The fact that the Higgs couplings are very much SM-like also holds for the effective mass matrix of the light (SM) fermions which is obtained after integrating out the heavy VL fermions, and we wish to show this analytically. The $4 \times 4$ mass matrix (13) can be rotated to a basis where there is a heavy $2 \times 2$ block. This is given by the transformation

$$
M^{\ell}=\left(X_{L}\right)^{\dagger} \mathcal{M}^{\ell} X_{R}
$$

with
$X_{L}=\left(\begin{array}{cccc}1 & 0 & 0 & 0 \\ 0 & N_{2 L} \lambda_{L} v_{\Phi} & N_{3 L} \lambda_{3} v_{\Phi} & N_{4 L} \lambda_{L} v_{\Phi} \\ 0 & N_{2 L} \lambda_{3} v_{\Phi} & -N_{3 L} \lambda_{L} v_{\Phi} & N_{4 L} \lambda_{3} v_{\Phi} \\ 0 & N_{2 L} \lambda_{2} v_{\varphi} & 0 & -N_{4 L}\left(\lambda_{L}^{2}+\lambda_{3}^{2}\right) v_{\Phi}\end{array}\right)$

and

$X_{R}=\left(\begin{array}{cccc}1 & 0 & 0 & 0 \\ 0 & N_{2 R} \lambda_{E} v_{\Phi} & N_{2 R} \lambda_{3} v_{\Phi} & N_{2 R} \lambda_{2} v_{\varphi} \\ 0 & N_{3 R} \lambda_{3} v_{\Phi} & -N_{3 R} \lambda_{E} v_{\Phi} & 0 \\ 0 & N_{4 R} \lambda_{E} v_{\Phi} & N_{4 R} \lambda_{3} v_{\Phi} & -N_{4 R}\left(\lambda_{E}^{2}+\lambda_{3}^{2}\right) v_{\Phi}\end{array}\right)$,

where

$N_{2 L(R)}:=\left[\left(\lambda_{L(E)}^{2}+\lambda_{3}^{2}\right) v_{\Phi}^{2}+\lambda_{2}^{2} v_{\varphi}^{2}\right]^{-1 / 2}$,

$N_{3 L(R)}:=\left[\left(\lambda_{L(E)}^{2}+\lambda_{3}^{2}\right) v_{\Phi}^{2}\right]^{-1 / 2}$,

$N_{4 L(R)}:=\left[\left(\lambda_{L(E)}^{2}+\lambda_{3}^{2}\right) \lambda_{2}^{2} v_{\varphi}^{2}+\left(\lambda_{L(E)}^{2}+\lambda_{3}^{2}\right)^{2} v_{\Phi}^{2}\right]^{-1 / 2}$.

In terms of $2 \times 2$ blocks $M^{\ell}$ is then given by

$$
M^{\ell}=\left(\begin{array}{cc}
M_{\text {heavy }} & V_{R} \\
V_{L} & M_{\text {light }}
\end{array}\right) .
$$

Upon integrating out the heavy states perturbatively we obtain the effective $2 \times 2$ mass matrix for the light states given by

$$
M_{\text {light }}^{\text {eff }}=M_{\text {light }}-V_{L} M_{\text {heavy }}^{-1} V_{R} \text {. }
$$

In particular one finds that the effective light masses are to very high accuracy linear in the Higgs VEV. There are higher order corrections in $v$ but they are numerically irrelevant. Thus when one diagonalizes the fermion mass matrix, one simultaneously diagonalizes the coupling of the Higgs field to fermions. Hence there are no significant flavor violating Higgs couplings.

\section{APPENDIX C: DETAILS OF $\delta a_{\mu}^{Z^{\prime}}$}

The leading order contribution to the muon $g-2$ arising from the $Z^{\prime}$ coupling to leptons as in (31) is given by (see e.g. $[29,51])$

$$
\begin{aligned}
\delta a_{\mu}^{Z^{\prime}}= & -g^{\prime 2} \frac{m_{\mu}^{2}}{8 \pi^{2} M_{Z^{\prime}}^{2}} \sum_{a}\left[\left(\left|\left[\hat{g}_{L}^{\ell}\right]_{4 a}\right|^{2}+\left|\left[\hat{g}_{R}^{\ell}\right]_{4 a}\right|^{2}\right) F\left(x_{a}\right)\right. \\
& \left.+\operatorname{Re}\left(\left[\hat{g}_{L}^{\ell}\right]_{4 a}\left[\hat{g}^{\ell, *}{ }_{R}\right]_{4 a}\right) \frac{m_{a}}{m_{\mu}} G\left(x_{a}\right)\right]
\end{aligned}
$$


where $a$ runs over all leptons with mass $m_{a}$ in the loop, $x_{a}:=\left(m_{a} / M_{Z^{\prime}}\right)^{2}$, and the loop functions

$$
\begin{aligned}
F(x):= & \left(5 x^{4}-14 x^{3}+39 x^{2}-38 x-18 x^{2} \ln x+8\right) / \\
& {\left[12(1-x)^{4}\right], } \\
G(x):= & \left(x^{3}+3 x-6 x \ln x-4\right) /\left[2(1-x)^{3}\right] .
\end{aligned}
$$

\section{APPENDIX D: DETAILS OF $\tau \rightarrow \mu \gamma$}

The leading order contribution to $\tau \rightarrow \mu \gamma$ arises from the flavor off-diagonal $Z^{\prime}$ couplings between $\tau-\mathrm{VL}$ and $\mu-\mathrm{VL}$. We have used the general results given in [57] to find the leading order contributions to the partial width

$$
\Gamma(\tau \rightarrow \mu \gamma)=\frac{\alpha g^{\prime 4}}{1024 \pi^{4}} \frac{m_{\tau}^{5}}{M_{Z^{\prime}}^{4}}\left(\left|\tilde{\sigma}_{L}\right|^{2}+\left|\tilde{\sigma}_{R}\right|^{2}\right),
$$

with

$$
\begin{aligned}
& \tilde{\sigma}_{L}=\sum_{a}\left(\left[\hat{g}_{L}^{\ell}\right]_{4 a}\left[\hat{g}_{L}^{\ell}\right]_{a 3} F\left(x_{a}\right)+\frac{m_{a}}{m_{\tau}}\left[\hat{g}_{L}^{\ell}\right]_{4 a}\left[\hat{g}_{R}^{\ell}\right]_{a 3} G\left(x_{a}\right)\right), \\
& \tilde{\sigma}_{R}=\sum_{a}\left(\left[\hat{g}_{R}^{\ell}\right]_{4 a}\left[\hat{g}_{R}^{\ell}\right]_{a 3} F\left(x_{a}\right)+\frac{m_{a}}{m_{\tau}}\left[\hat{g}_{R}^{\ell}\right]_{4 a}\left[\hat{g}_{L}^{\ell}\right]_{a 3} G\left(x_{a}\right)\right),
\end{aligned}
$$

where $a$ runs over all leptons with mass $m_{a}$ in the loop, $x_{a}:=\left(m_{a} / M_{Z^{\prime}}\right)^{2}$, and the loop functions are the same as in $(\mathrm{C} 2)$ and $\mathrm{C} 3$.

\section{APPENDIX E: $Z^{\prime}$ COUPLINGS AT THE BEST FIT POINT(S)}

Below we show the coupling matrices of $Z^{\prime}$ in the mass basis for our best fit point (I.). The analogous couplings for point (II.) are qualitatively the same.

$$
\begin{aligned}
\hat{g}_{L}^{e} & =\left(\begin{array}{cccc}
-0.2669 & 0.2798 & 0.01800 & 0.3425 \\
0.2798 & -0.2934 & -0.01890 & -0.3591 \\
0.01800 & -0.01890 & 0.9996 & -0.0100 \\
0.3425 & -0.3591 & -0.0100 & -0.4393
\end{array}\right), \quad(\mathrm{E} 1) \\
\hat{g}_{R}^{\ell} & =\left(\begin{array}{cccc}
-0.2674 & -0.2799 & -0.01800 & -0.3427 \\
-0.2799 & -0.2930 & -0.01890 & -0.3588 \\
-0.01800 & -0.01890 & 0.9996 & -0.0100 \\
-0.3427 & -0.3588 & -0.0100 & -0.4392
\end{array}\right), \quad(\mathrm{E} 2) \\
\hat{g}_{L}^{d} & =\left(\begin{array}{cccc}
-0.000100 & -0.004400 & 0.0007000 & 0.01030 \\
-0.004400 & -0.1539 & 0.02570 & 0.3613 \\
0.0007000 & 0.02570 & 0.9994 & -0.006300 \\
0.01030 & 0.3613 & -0.006300 & -0.8454
\end{array}\right),
\end{aligned}
$$

$$
\hat{g}_{R}^{d}=\left(\begin{array}{cccc}
-0.8418 & 0.05670 & 0.01670 & 0.3604 \\
0.05670 & -0.003800 & -0.001100 & -0.02430 \\
0.01670 & -0.001100 & 0.9998 & 5.546 \times 10^{-6} \\
0.3604 & -0.02430 & 5.546 \times 10^{-6} & -0.1542
\end{array}\right) \text {. }
$$

\section{APPENDIX F: Z COUPLINGS AT THE BEST FIT POINT(S)}

Below we show the coupling matrices of $Z$ in the mass basis for our best fit point (I.). The analogous couplings for point (II.) are qualitatively the same. The tree-level couplings of $Z$ to the SM families are very close to their SM values. For example, we find $\left|\left(\mathrm{W}_{\ell}\right)_{\mu \mu}-1\right| \lesssim 10^{-9} \ll 10^{-3}$ and $\left|\left(\mathrm{W}_{\mathrm{d}}\right)_{b s}\right| \lesssim 10^{-9} \ll 10^{-5}$ thereby easily fulfilling the bounds on these quantities as defined in [70].

$$
\begin{gathered}
\hat{g}_{L}^{Z, \ell}=\frac{g}{c_{W}}\left(\begin{array}{cccc}
-0.01592 & 0.2497 & 5.064 \times 10^{-6} & -2.580 \times 10^{-5} \\
0.2497 & -0.03948 & 4.831 \times 10^{-6} & -2.461 \times 10^{-5} \\
5.064 \times 10^{-6} & 4.831 \times 10^{-6} & -0.2777 & -4.990 \times 10^{-10} \\
-2.580 \times 10^{-5} & -2.461 \times 10^{-5} & -4.990 \times 10^{-10} & -0.2777
\end{array}\right), \\
\hat{g}_{R}^{Z, \ell}=\frac{g}{c_{W}}\left(\begin{array}{cccc}
-0.03910 & 0.2497 & 5.061 \times 10^{-6} & -2.578 \times 10^{-5} \\
0.2497 & -0.01640 & -4.835 \times 10^{-6} & 2.463 \times 10^{-5} \\
5.061 \times 10^{-6} & -4.835 \times 10^{-6} & 0.2223 & 4.991 \times 10^{-10} \\
-2.578 \times 10^{-5} & 2.463 \times 10^{-5} & 4.991 \times 10^{-10} & 0.2223
\end{array}\right),
\end{gathered}
$$




$$
\begin{gathered}
\hat{g}_{L}^{Z, d}=\frac{g}{c_{W}}\left(\begin{array}{cccc}
0.07369 & -0.01430 & -4.141 \times 10^{-6} & -4.387 \times 10^{-5} \\
-0.01430 & -0.4255 & 1.185 \times 10^{-7} & 1.255 \times 10^{-6} \\
-4.141 \times 10^{-6} & 1.185 \times 10^{-7} & -0.4259 & 3.636 \times 10^{-10} \\
-4.387 \times 10^{-5} & 1.255 \times 10^{-6} & 3.636 \times 10^{-10} & -0.4259
\end{array}\right), \\
\hat{g}_{R}^{Z, d}=\frac{g}{c_{W}}\left(\begin{array}{cccc}
0.07185 & -0.03344 & 1.568 \times 10^{-6} & 9.477 \times 10^{-6} \\
-0.03344 & -0.4237 & 2.334 \times 10^{-5} & 1.410 \times 10^{-4} \\
1.568 \times 10^{-6} & 2.334 \times 10^{-5} & 0.0741 & -6.614 \times 10^{-9} \\
9.477 \times 10^{-6} & 1.410 \times 10^{-4} & -6.614 \times 10^{-9} & 0.0741
\end{array}\right) .
\end{gathered}
$$

[1] G.W. Bennett et al. (Muon $g-2$ Collaboration), Final report of the Muon E821 anomalous magnetic moment measurement at BNL, Phys. Rev. D 73, 072003 (2006).

[2] C. Patrignani et al. (Particle Data Group), Review of particle physics, Chin. Phys. C 40, 100001 (2016).

[3] R. Aaij et al. (LHCb Collaboration), Differential branching fractions and isospin asymmetries of $B \rightarrow K^{(*)} \mu^{+} \mu^{-}$decays, J. High Energy Phys. 06 (2014) 133.

[4] R. Aaij et al. (LHCb Collaboration), Test of Lepton Universality Using $B^{+} \rightarrow K^{+} \ell^{+} \ell^{-}$Decays, Phys. Rev. Lett. 113, 151601 (2014).

[5] R. Aaij et al. (LHCb Collaboration), Differential branching fraction and angular analysis of the decay $B_{s}^{0} \rightarrow \phi \mu^{+} \mu^{-}$, J. High Energy Phys. 07 (2013) 084.

[6] J. P. Lees et al. (BABAR Collaboration), Measurement of the $B \rightarrow X_{s} l^{+} l^{-}$Branching Fraction and Search for Direct $C P$ Violation from a Sum of Exclusive Final States, Phys. Rev. Lett. 112, 211802 (2014).

[7] R. Aaij et al. (LHCb Collaboration), Angular analysis and differential branching fraction of the decay $B_{s}^{0} \rightarrow \phi \mu^{+} \mu^{-}$, J. High Energy Phys. 09 (2015) 179.

[8] R. Aaij et al. (LHCb Collaboration), Measurement of Form-Factor-Independent Observables in the Decay $B^{0} \rightarrow K^{* 0} \mu^{+} \mu^{-}$, Phys. Rev. Lett. 111, 191801 (2013).

[9] R. Aaij et al. (LHCb Collaboration), Angular analysis of the $B^{0} \rightarrow K^{* 0} \mu^{+} \mu^{-}$decay using $3 \mathrm{fb}^{-1}$ of integrated luminosity, J. High Energy Phys. 02 (2016) 104.

[10] CMS Collaboration, Measurement of the $P_{1}$ and $P_{5}^{\prime}$ angular parameters of the decay $\mathrm{B}^{0} \rightarrow \mathrm{K}^{* 0} \mu^{+} \mu^{-}$in proton-proton collisions at $\sqrt{s}=8 \mathrm{TeV}$, arXiv:1710.02846 [Phys. Lett. B (to be published)].

[11] V. Khachatryan et al. (CMS Collaboration), Angular analysis of the decay $B^{0} \rightarrow K^{* 0} \mu^{+} \mu^{-}$from $p p$ collisions at $\sqrt{s}=8 \mathrm{TeV}$, Phys. Lett. B 753, 424 (2016).

[12] A. Abdesselam et al. (Belle Collaboration), Angular analysis of $B^{0} \rightarrow K^{*}(892)^{0} \ell^{+} \ell^{-}$, arXiv:1604.04042.

[13] S. Wehle et al. (Belle Collaboration), Lepton-FlavorDependent Angular Analysis of $B \rightarrow K^{*} \ell^{+} \ell^{-}$, Phys. Rev. Lett. 118, 111801 (2017).
[14] ATLAS Collaboration, Angular analysis of $B_{d}^{0} \rightarrow K^{*} \mu^{+} \mu^{-}$ decays in $p p$ collisions at $\sqrt{s}=8 \mathrm{TeV}$ with the ATLAS detector.

[15] W. Altmannshofer, C. Niehoff, P. Stangl, and D. M. Straub, Status of the $B \rightarrow K^{*} \mu^{+} \mu^{-}$anomaly after Moriond 2017, Eur. Phys. J. C 77, 377 (2017).

[16] W. Altmannshofer, P. Stangl, and D. M. Straub, Interpreting hints for lepton flavor universality violation, Phys. Rev. D 96, 055008 (2017).

[17] A. K. Alok, B. Bhattacharya, A. Datta, D. Kumar, J. Kumar, and D. London, New physics in $b \rightarrow s \mu^{+} \mu^{-}$after the measurement of $R_{K^{*}}$, Phys. Rev. D 96, 095009 (2017).

[18] B. Capdevila, A. Crivellin, S. Descotes-Genon, J. Matias, and J. Virto, Patterns of new physics in $b \rightarrow s \ell^{+} \ell^{-}$ transitions in the light of recent data, J. High Energy Phys. 01 (2018) 093.

[19] M. Ciuchini, A. M. Coutinho, M. Fedele, E. Franco, A. Paul, L. Silvestrini, and M. Valli, On flavourful Easter eggs for new physics hunger and lepton flavour universality violation, Eur. Phys. J. C 77, 688 (2017).

[20] G. D’Amico, M. Nardecchia, P. Panci, F. Sannino, A. Strumia, R. Torre, and A. Urbano, Flavour anomalies after the $R_{K^{*}}$ measurement, J. High Energy Phys. 09 (2017) 010.

[21] L.-S. Geng, B. Grinstein, S. Jäger, J. Martin Camalich, X.-L. Ren, and R.-X. Shi, Towards the discovery of new physics with lepton-universality ratios of $b \rightarrow s \ell \ell$ decays, Phys. Rev. D 96, 093006 (2017).

[22] D. Ghosh, Explaining the $R_{K}$ and $R_{K^{*}}$ anomalies, Eur. Phys. J. C 77, 694 (2017).

[23] G. Hiller and I. Nisandzic, $R_{K}$ and $R_{K^{*}}$ beyond the standard model, Phys. Rev. D 96, 035003 (2017).

[24] A. J. Buras and M. Munz, Effective Hamiltonian for $B \rightarrow X_{s} e^{+} e^{-}$beyond leading logarithms in the NDR and HV schemes, Phys. Rev. D 52, 186 (1995).

[25] C. Bobeth, M. Misiak, and J. Urban, Photonic penguins at two loops and $m_{t}$ dependence of $B R\left[B \rightarrow X_{s} l^{+} l^{-}\right]$, Nucl. Phys. B574, 291 (2000).

[26] D. Bardhan, P. Byakti, and D. Ghosh, Role of tensor operators in $R_{K}$ and $R_{K^{*}}$, Phys. Lett. B 773, 505 (2017). 
[27] A. Czarnecki and W. J. Marciano, Muon anomalous magnetic moment: A harbinger for "new physics", Phys. Rev. D 64, 013014 (2001).

[28] K. Kannike, M. Raidal, D. M. Straub, and A. Strumia, Anthropic solution to the magnetic muon anomaly: The charged see-saw, J. High Energy Phys. 02 (2012) 106; Erratum, 10(2012) 136.

[29] R. Dermíšek and A. Raval, Explanation of the Muon $g-2$ anomaly with vectorlike leptons and its implications for Higgs decays, Phys. Rev. D 88, 013017 (2013).

[30] W. Altmannshofer, S. Gori, M. Pospelov, and I. Yavin, Quark flavor transitions in $L_{\mu}-L_{\tau}$ models, Phys. Rev. D 89, 095033 (2014).

[31] A. Crivellin, G. D'Ambrosio, and J. Heeck, Explaining $h \rightarrow \mu^{ \pm} \tau^{\mp}, B \rightarrow K^{*} \mu^{+} \mu^{-}$, and $B \rightarrow K \mu^{+} \mu^{-} / B \rightarrow K e^{+} e^{-}$in a Two-Higgs-Doublet Model with Gauged $L_{\mu}-L_{\tau}$, Phys. Rev. Lett. 114, 151801 (2015).

[32] S. F. King, Flavourful $Z^{\prime}$ models for $R_{K^{(*)}}$, J. High Energy Phys. 08 (2017) 019.

[33] B. Allanach, F. S. Queiroz, A. Strumia, and S. Sun, Z' models for the LHCb and $g-2$ muon anomalies, Phys. Rev. D 93, 055045 (2016); Erratum, 95, 119902 (2017).

[34] W. Altmannshofer, M. Carena, and A. Crivellin, $L_{\mu}-L_{\tau}$ theory of Higgs flavor violation and $(g-2)_{\mu}$, Phys. Rev. D 94, 095026 (2016).

[35] E. Megias, M. Quiros, and L. Salas, $g_{\mu}-2$ from vector-like leptons in warped space, J. High Energy Phys. 05 (2017) 016.

[36] T. Kobayashi, S. Raby, and R.-J. Zhang, Constructing 5-D orbifold grand unified theories from heterotic strings, Phys. Lett. B 593, 262 (2004).

[37] T. Kobayashi, S. Raby, and R.-J. Zhang, Searching for realistic 4d string models with a Pati-Salam symmetry: Orbifold grand unified theories from heterotic string compactification on a Z(6) orbifold, Nucl. Phys. B704, 3 (2005).

[38] W. Buchmuller, K. Hamaguchi, O. Lebedev, and M. Ratz, Supersymmetric Standard Model from the Heterotic String, Phys. Rev. Lett. 96, 121602 (2006).

[39] O. Lebedev, H. P. Nilles, S. Raby, S. Ramos-Sanchez, M. Ratz, P. K.S. Vaudrevange, and A. Wingerter, A minilandscape of exact MSSM spectra in heterotic orbifolds, Phys. Lett. B 645, 88 (2007).

[40] O. Lebedev, H. P. Nilles, S. Raby, S. Ramos-Sanchez, M. Ratz, P. K. S. Vaudrevange, and A. Wingerter, Heterotic road to the MSSM with $R$ parity, Phys. Rev. D 77, 046013 (2008).

[41] O. Lebedev, H. P. Nilles, S. Ramos-Sanchez, M. Ratz, and P. K. S. Vaudrevange, Heterotic mini-landscape. (II). Completing the search for MSSM vacua in a Z(6) orbifold, Phys. Lett. B 668, 331 (2008).

[42] M. Blaszczyk, S. Groot Nibbelink, M. Ratz, F. Ruehle, M. Trapletti, and P.K.S. Vaudrevange, A Z2xZ2 standard model, Phys. Lett. B 683, 340 (2010).

[43] R. Kappl, B. Petersen, S. Raby, M. Ratz, R. Schieren, and P. K. S. Vaudrevange, String-derived MSSM vacua with residual R symmetries, Nucl. Phys. B847, 325 (2011).

[44] T. Kobayashi, H. P. Nilles, F. Ploger, S. Raby, and M. Ratz, Stringy origin of non-Abelian discrete flavor symmetries, Nucl. Phys. B768, 135 (2007).
[45] P. Ko, T. Kobayashi, J.-h. Park, and S. Raby, String-derived D(4) flavor symmetry and phenomenological implications, Phys. Rev. D 76, 035005 (2007); Erratum, 76, 059901 (2007).

[46] B. Capdevila, A. Crivellin, S. Descotes-Genon, L. Hofer, and J. Matias, Searching for New Physics with $b \rightarrow s \tau^{+} \tau^{-}$ Processes, arXiv:1712.01919 [Phys. Rev. Lett. (to be published)].

[47] C. Bobeth and U. Haisch, New physics in $\Gamma_{12}^{s}:(\bar{s} b)(\bar{\tau} \tau)$ operators, Acta Phys. Pol. B 44, 127 (2013).

[48] J. F. Kamenik, S. Monteil, A. Semkiv, and L. V. Silva, Lepton polarization asymmetries in rare semi-tauonic $b \rightarrow s$ exclusive decays at FCC-ee, Eur. Phys. J. C 77, 701 (2017).

[49] A. J. Buras, J. Girrbach-Noe, C. Niehoff, and D. M. Straub, $B \rightarrow K^{(*)} \nu \bar{\nu}$ decays in the Standard Model and beyond, J. High Energy Phys. 02 (2015) 184.

[50] L. Calibbi, A. Crivellin, and T. Ota, Effective Field Theory Approach to $b \rightarrow s \ell \ell^{\left({ }^{\prime}\right)}, B \rightarrow K^{(*)} \nu \bar{\nu}$, and $B \rightarrow D^{(*)} \tau \nu$ with Third Generation Couplings, Phys. Rev. Lett. 115, 181801 (2015).

[51] F. Jegerlehner and A. Nyffeler, The Muon $g-2$, Phys. Rep. 477, 1 (2009).

[52] A. J. Buras, F. De Fazio, and J. Girrbach, The anatomy of Z' and $\mathrm{Z}$ with flavour changing neutral currents in the flavour precision era, J. High Energy Phys. 02 (2013) 116.

[53] L. Di Luzio, M. Kirk, and A. Lenz, One constraint to kill them all?, arXiv:1712.06572.

[54] W. Altmannshofer and D. M. Straub, New physics in $b \rightarrow s$ transitions after LHC run 1, Eur. Phys. J. C 75, 382 (2015).

[55] Y. Okada, K.-i. Okumura, and Y. Shimizu, $\vec{\mu} e \gamma$ and $\vec{\mu} 3 e$ processes with polarized muons and supersymmetric grand unified theories, Phys. Rev. D 61, 094001 (2000).

[56] Y. Kuno and Y. Okada, Muon decay and physics beyond the standard model, Rev. Mod. Phys. 73, 151 (2001).

[57] L. Lavoura, General formulae for $f_{1} \rightarrow f_{2} \gamma$, Eur. Phys. J. C 29, 191 (2003).

[58] J. Hisano, T. Moroi, K. Tobe, and M. Yamaguchi, Lepton flavor violation via right-handed neutrino Yukawa couplings in supersymmetric standard model, Phys. Rev. D 53, 2442 (1996).

[59] K. Ishiwata and M. B. Wise, Phenomenology of heavy vectorlike leptons, Phys. Rev. D 88, 055009 (2013).

[60] A. Abada, M. E. Krauss, W. Porod, F. Staub, A. Vicente, and C. Weiland, Lepton flavor violation in low-scale seesaw models: SUSY and non-SUSY contributions, J. High Energy Phys. 11 (2014) 048.

[61] W. Altmannshofer, S. Gori, M. Pospelov, and I. Yavin, Neutrino Trident Production: A Powerful Probe of New Physics with Neutrino Beams, Phys. Rev. Lett. 113, 091801 (2014).

[62] E. Fernandez-Martinez, J. Hernandez-Garcia, and J. LopezPavon, Global constraints on heavy neutrino mixing, J. High Energy Phys. 08 (2016) 033.

[63] S. Antusch and O. Fischer, Non-unitarity of the leptonic mixing matrix: Present bounds and future sensitivities, J. High Energy Phys. 10 (2014) 094.

[64] F. del Aguila, J. de Blas, and M. Perez-Victoria, Effects of new leptons in electroweak precision data, Phys. Rev. D 78, 013010 (2008). 
[65] A. Joglekar, P. Schwaller, and C. E. M. Wagner, Dark matter and enhanced Higgs to di-photon rate from vector-like leptons, J. High Energy Phys. 12 (2012) 064.

[66] J. Kearney, A. Pierce, and N. Weiner, Vectorlike fermions and Higgs couplings, Phys. Rev. D 86, 113005 (2012).

[67] S. Antusch and V. Maurer, Running quark and lepton parameters at various scales, J. High Energy Phys. 11 (2013) 115.

[68] G. Barenboim, F. J. Botella, and O. Vives, Constraining models with vector-like fermions from FCNC in $K$ and $B$ physics, Nucl. Phys. B613, 285 (2001).

[69] A. K. Alok, S. Banerjee, D. Kumar, and S. Uma Sankar, Flavor signatures of isosinglet vector-like down quark model, Nucl. Phys. B906, 321 (2016).

[70] F. J. Botella, G. C. Branco, and M. Nebot, Singlet heavy fermions as the origin of $\mathrm{B}$ anomalies in flavour changing neutral currents, arXiv:1712.04470.

[71] A. Falkowski, D. M. Straub, and A. Vicente, Vector-like leptons: Higgs decays and collider phenomenology, J. High Energy Phys. 05 (2014) 092.

[72] V. Khachatryan et al. (CMS Collaboration), Search for pairproduced vectorlike $B$ quarks in proton-proton collisions at $\sqrt{s}=8 \mathrm{TeV}$, Phys. Rev. D 93, 112009 (2016).

[73] B. Aubert et al. (BABAR Collaboration), Searches for Lepton Flavor Violation in the Decays $\tau^{+} \rightarrow e^{+} \gamma$ and $\tau^{+} \rightarrow \mu^{+} \gamma$, Phys. Rev. Lett. 104, 021802 (2010).

[74] K. Hayasaka et al., Search for lepton flavor violating tau decays into three leptons with 719 million produced tau + tau- pairs, Phys. Lett. B 687, 139 (2010).
[75] J. P. Lees et al. (BABAR Collaboration), Search for $B^{+} \rightarrow K^{+} \tau^{+} \tau^{-}$at the BABAR Experiment, Phys. Rev. Lett. 118, 031802 (2017).

[76] J. P. Lees et al. (BABAR Collaboration), Search for $B \rightarrow K^{(*)} \nu \bar{\nu}$ and invisible quarkonium decays, Phys. Rev. D 87, 112005 (2013).

[77] O. Lutz et al. (Belle Collaboration), Search for $B \rightarrow h^{(*)} \nu \bar{\nu}$ with the full Belle $\Upsilon(4 S)$ data sample, Phys. Rev. D 87, 111103 (2013).

[78] T. Aushev et al., Physics at Super B Factory, arXiv: 1002.5012 .

[79] V. Khachatryan et al. (CMS Collaboration), Search for narrow resonances in dilepton mass spectra in proton-proton collisions at $\sqrt{s}=13 \mathrm{TeV}$ and combination with $8 \mathrm{TeV}$ data, Phys. Lett. B 768, 57 (2017).

[80] M. Aaboud et al. (ATLAS Collaboration), Search for new high-mass phenomena in the dilepton final state using $36 \mathrm{fb}^{-1}$ of proton-proton collision data at $\sqrt{s}=13 \mathrm{TeV}$ with the ATLAS detector, J. High Energy Phys. 10 (2017) 182.

[81] R. Alonso, P. Cox, C. Han, and T. T. Yanagida, Flavoured $B-L$ local symmetry and anomalous rare $B$ decays, Phys. Lett. B 774, 643 (2017).

[82] C. Bonilla, T. Modak, R. Srivastava, and J. W. F. Valle, $U(1)_{B_{3}-3 L_{\mu}}$ gauge symmetry as the simplest description of $b \rightarrow s$ anomalies, arXiv:1705.00915.

[83] L. Bian, S.-M. Choi, Y.-J. Kang, and H. M. Lee, A minimal flavored $U(1)^{\prime}$ for $B$-meson anomalies, Phys. Rev. D 96, 075038 (2017). 